\title{
An Energy-Aware Engineered Control Plane for Wavelength-Routed Networks
}

\author{
Sergio Ricciardi ${ }^{1 *}$, Jiayuan Wang ${ }^{2}$, Francesco Palmieri ${ }^{3}$, Davide Careglio ${ }^{1}$, Lars Dittman ${ }^{2}$ \\ ${ }^{1}$ Department of Computer Architecture, Universitat Politècnica de Catalunya - BarcelonaTech (UPC), \\ C. Jordi Girona 31, E-08034, Barcelona. Spain, sergio.ricciardi@ac.upc.edu \\ ${ }^{2}$ Department of Photonics Engineering, Technical University of Denmark (DTU), \\ Orsteds Plads, DK-2800 Kgs. Lyngby, Denmark, jwan@fotonik.dtu.dk \\ ${ }^{3}$ Department of Industrial and Information Engineering, Second University of Naples (SUN), \\ Via Roma 29, I-81031 Aversa, Italy, francesco.palmieri@unina.it
}

\begin{abstract}
Despite the significant degree of technological maturity reached by large scale optical transmission infrastructures, new critical issues are emerging as side effects associated to their energy consumption, environmental impact and related costs, now accounting for an important part of the networks' operational expenditures. To face this problem, we propose a singlestage RWA scheme, based on several network engineering extensions to the GMPLS control plane protocols, mainly OSPF, with new composed metrics and information transport facilities. It is structured according to a simple multi-objective optimization approach aiming at containing the network's energy consumption and ecological impact while balancing the overall load and maintaining an acceptable connection demand satisfaction rate. The proposed solution is able to operate effectively in wide area wavelength routing scenarios, where multiple heterogeneous equipment, ranging from pure photonic to opaque routing/switching, amplification and regeneration devices are deployed in a GMPLS-empowered network. Copyright (c) 2012 John Wiley \& Sons, Ltd.
\end{abstract}

\section{* Correspondence}

Department of Computer Architecture, Universitat Politècnica de Catalunya - BarcelonaTech (UPC),

C. Jordi Girona 31, E-08034, Barcelona. Spain, sergio.ricciardi@ac.upc.edu

\section{INTRODUCTION}

New internet-based applications and converged data/multimedia services are quickly boosting the worldwide development of very high-speed next generation telecommunication infrastructures, and optical networking technologies, already supporting multi-terabit per second capacity, fast and flexible provisioning, as well as advanced QoS and traffic engineering capabilities, are widely recognized to be the basic building blocks for these infrastructures. In particular, by using Wavelength Division Multiplexing (WDM), multiple different channels, associated to individual wavelengths, can be carved on a single fiber where each channel can operate at its peak transport capability. This enables the realization of wavelength routed optical backbone architectures, empowering both metropolitan and wide area networking scenarios where the communication infrastructure is composed of wavelength switching devices connected by multiple fiber links. In these scenarios, despite the high degree of robustness and maturity reached by optical transmission technologies, there are still a lot of open issues associated to the energy consumption and green house gases (GHG) emissions of large scale transport infrastructures. In fact, energy costs account for an important part of the operational expenditures (OPEX) 
in modern transport networks, and, at the same time, additional taxes, introduced for environment safeguarding purposes, are paid in several countries according to the amount of emitted GHG (mainly $\mathrm{CO}_{2}$ ) in an effort to establish a carbon containment strategy such as the cap\&trade [1]. Clearly, while networking equipment does not directly emit GHG, these taxes are associated to the energy plants powering them, which do emit GHG in a quantity depending on the type of energy source exploited (e.g. oil, carbon, natural gas, biomasses, geothermal, etc.). The use of renewable, green energy sources (such as solar, wind, tide) represents part of the solution; however, due to their intermittent nature, legacy dirty energy sources (fossil fuels) are usually employed to guarantee the continuous supplying of power. In order to report some cases that better describe the phenomenon, in the UK, British Telecom is the largest single power consumer [2] whereas in Italy and in France, Telecom Italia and France Telecom respectively, are the second largest consumers of electricity after their National Railway systems, with about $2 T W h$ of energy per year [3, 4]. Consequently, energy efficiency and eco-sustainability are now among the major concerns for network operators, and we can easily foresee that, in the next few years, large-scale transport networks will be no longer constrained by their capacity and performance, but rather by their operational costs associated to energy consumption and environmental impact [5].

To face this challenge, it is essential to revise the next-generation network architectures and protocols with energy efficiency and eco-sustainability in mind, by introducing power and GHG awareness within the control plane framework in order to properly influence the routing and wavelength selection processes by privileging the use of renewable energy sources and energy efficient links and switching devices, in such a way that the overall power consumption and GHG emissions can be minimized. However, in order to maximize energy-efficiency, many of the actual solutions tend to disregard the resource usage optimization objectives that characterize traditional network management practices, whose basic tasks are keeping the traffic load distribution fairly balanced on the available links as well as routing the traffic on some "privileged" connections according to specific engineering policies. The apparently conflicting energy and traffic engineering-related goals may be almost straightforwardly harmonized by putting them together into a common traffic-engineering enabled control plane framework such as the Generalized Multi-Protocol Label Switching (GMPLS), providing advanced routing and signaling facilities that can be easily extended to support energy aware decisions within the traditional traffic engineering framework. In this work, this has been accomplished by proposing extensions to the Open Shortest Path First (OSPF-TE) link-state routing protocol to continuously convey devices' energy-related information throughout the network and determine routes, by using a shortest-path routing scheme based on dynamic link costs that are subject to both traffic engineering and power consumption constraints, also considering the specific kind of energy source (dirty or renewable) used for powering the traversed nodes. Most of this energy-related information is provided by specific interfaces and communication facilities offered by modern Smart Power Grid systems that in the near future will fully interact with all the transport networks and ICT infrastructures [6]. This gives origin to a multiobjective routing problem that can be solved though a classic weighted sum approach, aggregating the two objectives into a single one by assigning to each of them a specific cost function so that the cost of each edge used in shortest (or better, minimum cost) path calculation results from a linear combination of the individual functions. Clearly, the most probable outcome would be that some of the selected minimum costs paths would not be the best ones neither in terms of traffic distribution nor in terms of energy consumption containment, but the associated power savings and ecological footprint improvements would be appreciable without adversely affecting in a significant way the traditional network operations.

The effectiveness of the proposed scheme has been demonstrated by performing extensive simulation experiments, resulting in an overall reduction of network-wide energy demand and GHG emissions that was extremely satisfactory at the expense of a limited additional effort, due to the dynamic nature of the costs, and without losses in the perceivable network performance/behavior. Starting from this experience we envision that the proposed approach can foster more careful and complete routing strategies with the potential of driving the change towards a new generation of energy-efficient and eco-sustainable network infrastructures. 


\section{RELATED WORK}

Evolving towards greener and cheaper networks, in terms of both energy efficiency and eco-sustainability, is a really strong emerging trend widely exploited in recent networking research efforts. One of the first proposals, envisioning the concept of energy awareness in IP-based infrastructures has been presented in [7]. Starting from this experience, many other works focused on containing power consumption and $\mathrm{CO}_{2}$ emissions in communication networks, focusing on the impact of network parameters on the design of energy-aware protocols [8] and on the trade-offs between QoE and energy efficiency [9]. A very common approach relies on the so called sleep modes, based on temporarily switching off idle nodes or interfaces/line cards during low-demand hours [10, 11, 12]. Similarly, the ideas presented in [13] exploit the energy savings that can be achieved by dynamically adapting the network topology to its current load by introducing mathematical programming models that results in energy optimal topologies for given traffic conditions. On the other hand, the work presented in [14] proposes a new distributed routing protocol based on OSPF that, when the network is lightly loaded, routes the traffic through a minimal subset of nodes, putting unnecessary devices into sleep mode and hence reducing power consumption and GHG emissions. Analogously, the approach presented in [15] exploits node hibernation within the GMPLS framework, in order to contain power consumption in optical networks. The works presented in $[16,17]$ envision an eco-sustainable WDM network using renewable sources as much as possible by achieving minimization of dirty sources usage, and hence $\mathrm{CO}_{2}$ emissions, through a Linear Programming model. Other mathematical formulations based on integer linear programming are presented in [18] and [19], with the double objective of reducing both the energy consumption and the GHG emissions of network infrastructure by using multiple green and dirty energy sources and different objective functions. Since these formulations can be used to find an optimum solution of the offline Routing and Wavelength Assignment (RWA) problem, they give an upper bound for energy and GHG savings. Other interesting routing proposals exploiting the use of multiple power sources and containing GHG emissions are reported respectively in [20] and [21]. The work presented in [22] exploits with a dynamic multi-stage approach the minimization of both energy consumption and carbon footprint in WDM networks providing adaptive sub-wavelength routing facilities. In [23], energy savings in backbone networks is achieved through joint optimization of data placement and traffic routing. Most of the above approaches are characterized by multistage complex decision mechanisms or a quite limited dynamism, and hence are not easily and seamlessly applicable in a fully adaptive online scenario, often using power containment techniques based on switching down inactive elements. In modern fully dynamic on-line RWA approaches, sleep modes are not considered as practically affordable, for both performance and investment protection reasons [19]. Accordingly, the proposed shortest pathbased approach is mainly targeted at the introduction of new energy related facilities into standard GMPLSbased networks, by providing fully dynamic and adaptive RWA support, with minimal modification efforts and no performance impacts in the control plane protocols by ensuring, as possible, backward compatibility with all the existing implementations.

\section{ROUTING AND WAVELENGTH ASSIGNMENT IN THE GMPLS CONTROL PLANE}

GMPLS is currently recognized as the unified control plane of choice for optical wavelength switched networks operating according to a traditional connection oriented paradigm. It enables the setup and management of multiple end-to-end optical channels often referred as "lightpaths", carved on specific wavelengths on the physical network infrastructure, that pass exclusively through pure photonic switching devices. Such devices toggle wavelengths between a pair of source and destination ports without the need of any kind of optical-electronic conversions. The setup of a lightpath between two nodes implies the configuration of all the intermediate wavelength switching devices to connect an input port to an output one, by toggling a unique wavelength between them. In the most common transparent optical scenarios, where switching nodes do not provide wavelength conversion capability, the same wavelength must be used throughout the entire lightpath according to the so-called "continuity constraint". Hence, the process of establishing a new 
lightpath requires the selection of the shortest or minimum cost route between the involved endpoints together with a specific wavelength available on the entire route. Due to the limited number of choices available for path and wavelength selection, this process is associated to a well-known NP-complete problem, usually referred as RWA. Accordingly, when the network size and traffic load increases, computational burden becomes a real concern and the establishment of each new lightpath may potentially take an unacceptably long time, leading to delays or failures or in serving end-to-end connection requests and resulting in a potential degradation in the overall network performance. When the traffic matrix is known in advance the RWA problem may be effectively solved offline, but most of real world situations require dynamic and adaptive solutions, where the path and the wavelength to be used for carrying traffic from a source to a destination node is chosen dynamically, depending on the network link states. To simplify the process of dynamically finding a solution, the whole RWA problem can be partitioned into two independent sub-problems: spatial routing, based on the use of adaptive routing protocols and algorithms and spectral routing based on the use of specific heuristics for wavelength assignment.

In detail, within the GMPLS framework, several link state protocols, such as OSPF [24] or IS-IS [24], properly enhanced by introducing traffic engineering capabilities in a circuit switched scenario, are used to dynamically exchange link costs, features and status information, in order to provide to all the optical switching nodes a complete view of the network topology and of the available communication resources. Starting from this view, a Dijkstra-like adaptive shortest path routing algorithm (where the path length is the the sum of its links costs) is used to determine a minimum cost spatial route, based on variable communication link costs, continuously updated according to the link status information received. By properly setting the link costs (and hence the link weighting function), several traffic or network engineering objectives, such as efficient resource utilization, energy costs containment etc. can be simply and effectively achieved. Once a feasible best path is determined, an available wavelength must be seized an all its component links by using an heuristic technique such as random selection, First or Best Fit, in order to complete the lightpath setup. In absence of wavelength conversion capability in intermediate nodes, the continuity constraint must be enforced and the same wavelength has to be used on all the links. This introduces further complexity in the whole RWA scheme since, in order to ensure the same wavelength usage restriction, each switching node needs to be aware of the wavelength assignment plan of the entire network to be able of selecting routes that guarantee at least a common available wavelength on each link. Once the involved path and wavelengths are determined by the shortest path routing algorithm, a distributed signaling protocol, such as RSVP-TE [25] or CR-LDP [26] in the GMPLS control plane framework, is used to reserve the selected downstream and upstream wavelengths on each link along the specified route. Both protocols operate on a hop-by-hop basis, with RSVP-TE reserving resources in the backward direction (destinationinitiated reservation), and CR-LDP reserving resources in the forward direction (source-initiated reservation). Such signaling procedure has the main task of fully automating the lightpath establishment activity and, while these protocols are mainly used to reserve a wavelength determined at the path selection time, they may also be enhanced to introduce wavelength selection within the reservation process.

It can be easily observed that most of the energy-related (and, in general, equipment or link cost-constrained) optimization efforts within the RWA framework are concentrated on the spatial routing sub-problem, where the main decisions concerning the paths, and hence the involved equipment (with its energy consumption features) are taken. On the other hand, all the complex wavelengthassignment activities associated to spectral routing, once the necessary routing information is available, can have a very limited effect on the above optimization goals and thus can be handled straightforwardly through simple and effective heuristics, introducing some degree of enhancement in the computational efficiency and affecting only marginally the overall network management objectives. For this reason, most of the proposed constrained RWA approaches, aiming at achieving performance improvements or cost containment goals, mainly operate at the spatial routing level by using low complexity shortest path routing schemes relying on the determination of proper link cost models and weighting functions. 


\section{ENERGY CONSUMPTION IN WAVELENGTH-ROUTED NETWORKS}

The continuously growing bandwidth demands and connection volumes characterizing current network activities introduced a significant increment in the energy consumption of network devices, with obvious consequences on the operating costs of large-scale communication infrastructures. Clearly, the various devices used as the fundamental building blocks for realizing the typical wide area network infrastructures do not contribute to the overall power consumption in the same way. Electronic or opaque devices, such as routers or opto-electronics switches, usually operating on the network edge, are the highest power consumers, followed by $3 \mathrm{R}$ regenerators and ROADMs, whereas the transparent devices working entirely in the photonics domain such as OXCs (optical cross connects) and OAs (optical amplifiers) are characterized by a significantly lower power consumption. More precisely, all the switching and signal regeneration activities involving electronic conversion are very expensive both in terms of power dissipation due to the effect of losses during the transfer of electric charges caused by imperfect conductors and electrical isolators. The amount of energy required depends on several fabrication features (such the operating voltage, transition frequency, number of gates involved etc.). On the other hand, pure photonic communication technology requires very low energy for the transmission of signals, consisting essentially of light pulses, over the optical media. However, some electronic processing points may be still necessary, for traffic aggregation, control and monitoring as well as for signal regeneration purposes. These points should be avoided as much as possible in planning, designing and managing any component or sub-system within the communication infrastructure, since whenever the optical signal needs to be converted back and forth into the electronic domain the energy demand will remain remarkably high.

Since, until now, very limited efforts have been done to introduce energy awareness into the development of network devices, the power requirements of most of the traditional electronic interfaces and transmission/switching equipment are almost flat and hence independent from the equipment operational load, reaching huge amounts of Watts in high-end modular routing/switching nodes and large multi-chassis configurations [27][28]. However, the new generation of photonic networking components, typically designed with energy-efficiency concepts and principles in mind, will be characterized by a more careful management of their own consumption, resulting into different power demands in processing different types and amounts/rates of traffic [29]. That is, their consumption will scale with the traffic speeds and/or volumes processed and also depend on the type of processing required (i.e. fully transparent pass-through or opaque opto-electronic processing due to grooming, wavelength conversion, etc.). Some reference estimations of the fixed and variable power consumption values characterizing the most common network components in an MPLS-empowered optical network are reported in [27] and [30].

Starting from the above considerations, different RWA strategies can result in a very different energy consumption in the overall network, since the load can be balanced to keep the traffic volumes on individual interfaces or nodes under specific thresholds. This can lead to a reduced energy demand regimen, and analogously, lightpaths and logical topologies can be arranged in order to minimize the usage of energy-hungry devices, such as regenerators, wavelength converters, etc. Consequently, energy-awareness can assume an extreme importance in the RWA decision process, resulting in additional goals and constraints that must be considered in a more effective multi-objective optimization view of the entire RWA framework, that has to take into account the trade-offs between network resources usage (taking the best revenues from the initial investment/CAPEX), and power savings as well as environmental impacts (reducing GHG emissions and energy-related bills/taxes and hence OPEX).

\section{MODELING THE MULTI-OBJECTIVE RWA SCHEME}

A generic wavelength-routed network can be modeled as an undirected multi-graph $G=(V, E)$, with the nodes in $V$ being optical wavelength switches (OXC) or edge optical routers (OSR), and the edges in $E$ representing a physical connections between node pairs. OXC nodes provide photonic switching capabilities within the network core, i.e., switching a wavelength from an input fiber/interface to an output one, whereas OSRs are equipped with opaque switching matrices and 
Opto-Electronic converters to terminate wavelength-based circuits on the network edge.

Each node $v \in V$ is characterized by a specific fixed power consumption factor $\varphi_{v}$ (in Watts, W), required to keep the device powered on, and a variable power scaling factor $\varepsilon_{v}$ (in $\mathrm{W} / \mathrm{Gbps}$ ), modeling the energy required to support one unit of traffic flow (i.e., $1 \mathrm{Gbps}$ ). Thus, the power required by a node $v$ traversed by a connection with bit rate $b$, is given by $\varphi_{v}+b \cdot \varepsilon_{v}$. Note that per-node sleep mode is not allowed, due to the loss in connectivity degree, and to the significant investments involved in the acquisition of long-range fiber strands as well as sophisticated (and expensive) optical interfaces installed on high-end nodes with plenty of fiber connections attached.

From the ecological footprint perspective, the GHG emissions depending on the different energy sources that can supply a node $v$, can be modeled by using an emission factor $E_{v}$, expressed in grams of $\mathrm{CO}_{2} / \mathrm{kWh}$, so that by multiplying it for the power consumption during the operational time we obtain an estimation of the emitted $\mathrm{CO}_{2}$. Nevertheless, not all the energy sources are always available, since some of them, specifically most of the renewable ones, are strictly related to natural phenomena such as sunlight, wind and tide, and hence affected from periodic or unpredictable oscillations. To model this phenomenon, each device in the network is randomly assigned to an emission factor that may vary over time (e.g., the night-day cycle, high-low tides, etc.).

Each edge $(u, v) \in E$ is composed of a single wavelength division multiplexed fiber, supporting a maximum number $\lambda_{u v}^{\max }$ of channels, where $\lambda_{u v}^{\text {busy }} \leq \lambda_{u v}^{\max }$ of them are currently used. Depending on its length and transmission properties, a variable number $A_{u v}$ of optical amplifiers (typically one each $80 \mathrm{~km}$ ) may be present on the link $(u, v)$. We assume that all the amplifiers on the same link $(u, v)$ consume the same fixed amount of power $\varphi_{u, v}^{O A}$, and share the same energy source characterized by an emission factor $E_{u v}^{O A}$. No variable power consumption is modeled for OAs, since an OA amplifies the whole Cband (i.e., all the wavelengths at the same time); therefore, when calculating the network level power consumption, the energy consumption of the OAs on the link $(u, v)$ will be $\varphi_{u, v}^{O A}$ if the link $(u, v)$ is used by one or more wavelengths, otherwise it will be zero (OAs not used). As a consequence, when considering the individual link cost to be used in the lightpath construction process (i.e., the incremental cost of using the link in the Dijkstra algorithm), the OAs power consumption will be zero if the link is currently traversed by one or more lightpaths (since the OAs on the link $(u, v)$ are already active), otherwise their power consumption will be $\varphi_{u, v}^{O A}$ (since the OAs have to be activated for the first time).

Analogously, for very long fiber links, there can be a certain number $R_{u v}$ of intermediate $3 \mathrm{R}$ regeneration points (one each $1000 \mathrm{~km}$ ) characterized by fixed and load-dependent power consumption, respectively $\varphi_{u, v}^{3 R}$ (expressed in $\mathrm{W}$ ) and $\varepsilon_{u, v}^{3 R}$ (expressed in W/Gbps). Since $3 R$ regenerators operate per-channel, one $3 R$ is necessary for each wavelength of the link $(u, v)$, consuming $\varepsilon_{u, v}^{3 R}$ $\mathrm{W}$ for each Gbps of traffic. As for amplifiers, we assume that all the $3 \mathrm{R}$ regeneration points on a single link $(u, v)$ share the same energy source, whose ecological footprint is modeled by using the emission factor $E_{u v}^{3 R}$.

Given a traffic matrix describing all the connection requests to be served by the network, the basic goal of an RWA algorithm is the construction of a logical topology, realized by a set of dedicated communication channels, implemented through wavelength-based virtual circuits carved upon the network, that satisfy all the request by exhausting the minimum number of fiber links on the network graph and reducing, as possible, both the overall energy consumption/GHG emissions as well as the network usage unbalancing, such that the maximum number of requests can be simultaneously satisfied.

Each connection may be in theory characterized by multiple requirements such as optical link transmission properties (e.g. maximum available capacity, distance/delay, etc.) and impairments (e.g. bit-error rate, intermediate amplification steps) as well as energy related ones (e.g. maximum power drawn or type of energy sources at the endpoints, etc.). Such requirements become specific constraints for the routing algorithm that will work on a graph whose links or nodes are restricted to those ones in the original network topology that are fully compliant to the end-toend requirements of the connection to be routed. However, without losing generality, we can model connection requests with only a single constraint on the required bandwidth, and rely on the incorporation of all the other requirements within a bandwidth-routing scheme based on several QoS and impairment metrics transformed into a bandwidth requirement as shown in [31]. Therefore, the 
SPF algorithm is constrained by the available bandwidth and by the wavelength continuity constraint.

We assume that each connection has to be served with a bidirectional communication channel, transporting sets of traffic flows that cannot be split between multiple (different) paths. Thus, a connection can be routed on one or more (concatenated) fiber links between its source and destination nodes that provide available wavelength capacity, resulting into a dedicated logical circuit (lightpath) built upon a single wavelength. Consequently, allocation of wavelengths on the optical links on a lightpath takes place according to a circuit switched model where the same wavelength is assigned to each circuit for its entire lifetime. By using pure photonic devices such as amplifiers and transparent wavelength switches, lightpaths can span more than one fiber strand, remaining entirely optical from end to end. The use of any intermediate regeneration device converting the signals from the optical to the electronic domain and vice-versa is admitted only when strictly necessary, i.e., in presence of very long distance (more than $1000 \mathrm{~km}$ ) fiber links. Although intermediate regenerators allow wavelength conversion, wavelength continuity constraint is enforced in this model due to the economic, spatial, and efficiency problems affecting the conversion/reuse problem.

\subsection{A single-stage fully distributed approach}

In this scenario, we present an integrated singlestage energy-aware RWA algorithm based on a multiconstrained Shortest Path First (SPF) routing algorithm whose fundamental goal is determining, for each connection request characterized by a peak sustainable bit-rate $b$ (the committed bandwidth), the minimum cost (shortest) path based on a properly crafted perlink cost function $c_{u v}(b)$, dynamically associating a cost to each transmission link, that simultaneously considers (by assigning them a proper weight) the load balancing objectives, as well as the energy consumption and GHG emissions containment ones according to a multiobjective optimization approach based on scalarization. Consequently, the cost function results from a linear combination of the individual link costs associated to the above three independent objectives each one with its own weight, representing the relative importance of the individual objective into the overall optimization problem:

$$
\begin{array}{r}
c_{u v}(b)=\alpha_{P W R} \cdot c_{u v}^{P W R}(b)+\alpha_{G H G} \cdot c_{u v}^{G H G}(b)+ \\
+\alpha_{L B} \cdot c_{u v}^{L B},
\end{array}
$$

where $c_{u v}^{P W R}(b), c_{u v}^{G H G}(b)$ and $c_{u v}^{L B}$ are the individual costs related respectively to power consumption, GHG emission and capacity usage (in terms of wavelength occupancy) for the link $(u, v)$. The parameters $\alpha_{P W R}$, $\alpha_{G H G}$ and $\alpha_{L B}$, assuming values in the range $[0,1]$, define the relative importance (i.e., the scalarization weight) of the associated power consumption and GHG emission containment or load balancing goals within the whole multi-objective optimization problem. Clearly, the following constraint must be satisfied [32]:

$$
\alpha_{P W R}+\alpha_{G H G}+\alpha_{L B}=1 .
$$

In this way, a proper choice of the weights may drive the whole RWA decision process towards a pure energy consumption or GHG containment strategy or implement a balanced solution between the energy-related objectives and the efficient usage of the network traffic capacities.

The first component $\left(c_{u v}^{P W R}\right)$ of the cost function of eq. (1), associated to the power consumption of the link $(u, v)$, taxes a link according to the power consumption of the involved devices (extreme nodes $u, v$ and the OAs and 3Rs on the link), and depends on the committed bandwidth $b$ of the connection:

$$
\begin{aligned}
c_{u v}^{P W R} & =\left(\varphi_{u}+\varepsilon_{u} \cdot b\right)+\left(\varphi_{v}+\varepsilon_{v} \cdot b\right)+ \\
& +\left(\varphi_{u, v}^{O A} \cdot A_{u v}\right)+\left(\varphi_{u, v}^{3 R}+\varepsilon_{u, v}^{3 R} \cdot b\right) \cdot R_{u v},
\end{aligned}
$$

We recall that, since the edge cost function $c_{u v}^{P W R}$ is used when calculating the incremental link cost in the Dijkstra algorithm, $\varphi_{u, v}^{O A}$ affects the link cost only if there are no other lightpaths traversing the link $(u, v)$, otherwise its value will be zero.

Analogously, the second component $\left(c_{u v}^{G H G}\right)$, associated to the ecological footprint, taxes a link according to the GHG emission of its terminating nodes and intermediate amplifier or regenerator devices, and it is obtained by eq. (3) by adding the GHG emission factors, since here we are interested in its GHG emissions: 


$$
\begin{gathered}
c_{u v}^{G H G}=\left(\varphi_{u}+\varepsilon_{u} \cdot b\right) \cdot E_{u}+\left(\varphi_{v}+\varepsilon_{v} \cdot b\right) \cdot E_{v}+ \\
+\left(\varphi_{u, v}^{O A} \cdot A_{u v}\right) \cdot E_{u v}^{O A}+\left(\varphi_{u, v}^{3 R}+\varepsilon_{u, v}^{3 R} \cdot b\right) \cdot R_{u v} \cdot E_{u v}^{3 R} .
\end{gathered}
$$

Last, the cost component associated to wavelength occupancy $\left(c_{u v}^{L B}\right)$, independent from the requested connection's bandwidth, and defined by:

$$
c_{u v}^{L B}=\frac{\lambda_{u v}^{b u s y}}{\lambda_{u v}^{\max }}
$$

ensures a balanced usage of all the available network communication resources, in order to avoid the saturation of some more critical links that risk to become real bottlenecks in the overall network infrastructure. Note that the more a link is "dirty" (in terms of energy source), power-hungry or loaded, the higher its cost is.

The constrained SPF algorithm based on the above cost function will operate online in a fully decentralized way, that is, each node independently takes its routing and wavelength assignment decisions by running at each request of a dedicated end-to-end connection with specific bandwidth capacity requirements, with the goal of minimizing the cost of each connection, defined by the sum of the costs of each traversed link and determined according to the above per-link weighting function.

The convergence of all the autonomous decisions taken by the nodes towards a common RWA solution is ensured by the presence of a unique distributed control plane framework globally providing information associated to network topology and resources (link status/load and available wavelengths) as well as to energy demand (individual device consumption) and ecological footprint (available green or dirty energy source etc.). In such a way all the participating nodes will share the same network topology and status view, resulting in identical decisions when running the constrained SPF algorithm at the same time.

\subsection{Information Dissemination mechanisms}

In order to synchronize network views on all the nodes, a flexible and effective transport facility is needed to convey all the aforementioned enhanced link and node status information throughout the network. Analogously, an inter-node signaling mechanism is needed for the wavelength reservation and the establishment of paths. Specifically, in the GMPLS control plane framework, such function can be provided by properly extending the available link state routing protocols, such as OSPF, by introducing the needed energy-related information in the Traffic Engineering Link State Advertisements (TE-LSA) messages. These facilities are also already used in the GMPLS environment to provide wavelength availability information on all the links in order to associate wavelength selection to the path determination phase according to a specific policy (e.g., random, first or best fit).

In detail, to propagate throughout the network the information needed in the cost function of eq. (1), some extensions to the OSPF-TE link state routing protocol [33] are necessary. Opaque Link State Advertisements [34] (LSAs) for the OSPF protocol are used for implementing the proposed extensions. More precisely, Traffic Engineering LSAs with opaque type $=10$, having an intra-area flooding scope, are used as the transport mechanism for energy related information.

The protocol information carried by an opaque LSA is structured into extensible triplets known as Type-LengthValues (TLV). Each TLV consists of three fields: the first one is the TLV type, a 16-bits application-specific code, describing the kind of information being transported. The second field carries the length in of octets (or bytes) of the following variable-sized field, the value, containing the real data to be transported in the advertisement message. TLV structures can be nested, i.e. a TLV could carry another TLV known as sub-tlv. The OSPF-TE specification defines two top TLVs, namely the Router Address TLV and the Link TLV. The former one transports an address directly associated to the node connectivity, and the latter on can be characterized by 9 sub-TLVs describing the link traffic engineering features of interest. The detailed explanation of each field of the TE LSAs can be found in [35]. In our implementation, new sub-TLVs are added directly to the Router Address and Link TLVs into TELSAs. These LSA can describe properties associated to routers and point-to-point links, such as the power consumption or GHG emission factors for both nodes and amplifiers/regenerators. To transport the different information several sub-TLVs with Types in the range 32768-32777 (reserved for experimental applications) 
Table I. Sub-TLVs for TE LSA.

\begin{tabular}{c|c|c}
\hline Type & Length & Value \\
\hline \hline 32768 & 4 octets & Power Consumption Factor \\
\hline 32769 & 4 octets & $\mathrm{CO}_{2}$ Emission Factor \\
\hline 32770 & 4 octets & Wavelength Availability \\
\hline
\end{tabular}

have been used in both the Link TLV and Router TLV LSAs (see Tab. I for details). Therefore, in presence of a modification in the energy-related information of a network device, the involved nodes flood a TE-LSA throughout the network in order to propagate this change for being used by the Constrained SPF algorithm on all the nodes participating to the distributed RWA process. The proposed TE LSAs will be also flooded over the whole network on a fixed time-basis, disseminating all the needed energy-related source information in order to force a periodic re-synchronization.

The same mechanism can be used to describe the status of the wavelengths contained within a fiber link, transported within a specific available wavelength information sub-tlv of the Link TLV. Therefore, when an energy status changes, the corresponding terminating nodes broadcast a TE-LSA to disseminate this change to all the nodes in the network, so that the new status can be considered in the RWA decision process.

\section{EXPERIMENTAL EVALUATION AND RESULTS DISCUSSION}

The constrained SPF scheme with the proposed OSPFTE extension have been implemented in the OPNET [36] event-driven network simulator. Wavelength selection has been implemented according to a First-Fit policy. All the experiments have been performed on two different case study topologies: the European COST266 network [37], with 37 nodes and 55 bidirectional links (see Fig. 1), and the US NSFNet (see Fig. 2) with 14 nodes and 20 bidirectional links, both with 160 wavelengths per link (80 for each fiber, in order to simulate state-of-the-art high-end WDM systems).

Optical amplifiers and 3R regenerators are placed on each link at fixed distances, respectively every 80 and 1000 $\mathrm{Km}$. We enforce the wavelength continuity constraint and do not consider wavelength conversion at the regeneration points. As stated in Section 5, this is to limit the control
Table II. Fixed and variable power consumption for network devices

\begin{tabular}{clcc}
\hline \multicolumn{2}{c}{$\begin{array}{c}\text { Network Device } \\
\text { Type }\end{array}$} & \multicolumn{2}{c}{ Power Consumption } \\
Fixed (W) & Variable (W/Gbps) \\
\hline \hline \multirow{3}{*}{ OSR node } & low end & 285 & 4.5 \\
& medium end & 550 & 3 \\
& high end & 785 & 1.5 \\
\hline \multirow{3}{*}{ OXC node } & low end & 215 & 0.03 \\
& medium end & 445 & 0.02 \\
& high end & 685 & 0.01 \\
\hline Optical Amplifier & high end & 15 & n.a. \\
\hline 3R Regenerator & high end & 285 & 3 \\
\hline
\end{tabular}

plane complexity and functionalities. However, such an assumption does not affect the results since no blocking can happen in our simulative setup characterized by a strong resource overprovisioning degree. The power demands of OSR and OXC devices, mainly depending on their market segment, together with the ones associated to amplifiers and regenerators, are reported in Table II. Since our model does not allow per-node sleep mode (powering on and off entire network nodes), their fixed power demand values are irrelevant (they do not influence the simulation experiments). However we report them in the aforementioned table for model's completeness sake. On the other hand, the GHG emission associated to the energy sources powering the network devices are calculated by combining the power consumption data in Tab. II with the dynamic GHG emission factors reported in Tab. III.

Table III. Mean energy sources emissions [38]

\begin{tabular}{c|c}
\hline Energy source & $\begin{array}{c}\text { Mean } \mathrm{CO}_{2} \text { emissions } \\
\text { (grams per kWh) }\end{array}$ \\
\hline \hline Solar, Wind, Tide, Hydro-electrical & 0 \\
\hline Nuclear & 20 \\
\hline Geothermal & 107 \\
\hline Biomasses & 180 \\
\hline Natural gas & 370 \\
\hline Fuel & 880 \\
\hline Coal & 980 \\
\hline
\end{tabular}

Label Switched Path (LSP) connection requests, each with a maximum sustainable bit rate of $1 \mathrm{Gbps}$, to be routed on an individual wavelength, are generated as a Poisson process with exponentially distributed connection duration. This is the typical choice to model arrivals in a circuit switched telecommunications scenario [39]. Simulation results are generated with $95 \%$ confidence interval. The mean value of the connections lifetime $T$ has been set to $1,3,6,12$ and 24 hours (fixed in 


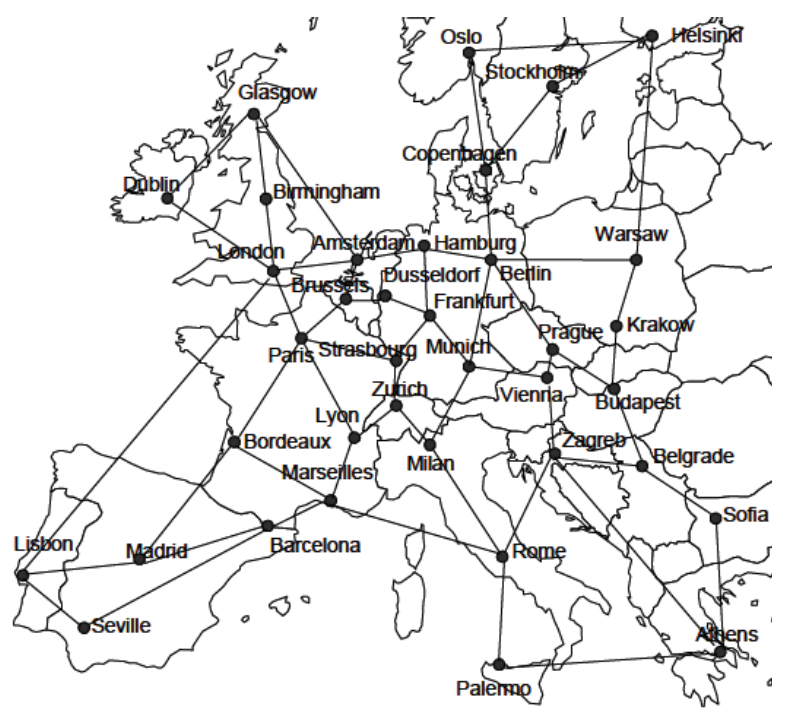

Figure 1. The Cost266 network.

each experiment), with mean inter-arrival time $\lambda=30$ minutes. The mean traffic load $L$ of a stable functioning system, given by Little's Law from queuing theory and obtained by the product of the average connection duration $T$ and average arrival rate $\lambda$ of connections, is maintained at a fixed value of $L=\lambda T=12$ Erlangs per node, for both the topologies reported in figures 1 and 2. This fixed load value has been properly chosen to progressively stress the network without introducing blocking of connections, in order to better observe the behavior of the proposed scheme in terms of power drawn and GHG emissions containment, without the adverse effects caused by blocking. At fixed time intervals (1, 3, 6, 12 and 24 hours), the energy sources randomly change, by assuming the values reported in Table III, and a new update of the power source information (TE LSA) is originated and flooded between neighbors using the proposed OSPFTE extension. This random change strategy has been introduced to mimic the intermittent availability of most of the renewable power sources (e.g., wind, direct sunlight with or without the adverse effect of clouds, ocean waves). The new carbon footprint information is updated in each LSA database, and thus, used for routing calculation. Simulation run time was set to 30 days, during which the power consumption, GHG emissions and hop count of the connections were monitored.

The best way to initially analyze the effectiveness of the individual objectives is to evaluate them separately, when only one optimization objective is pursued at a time and no

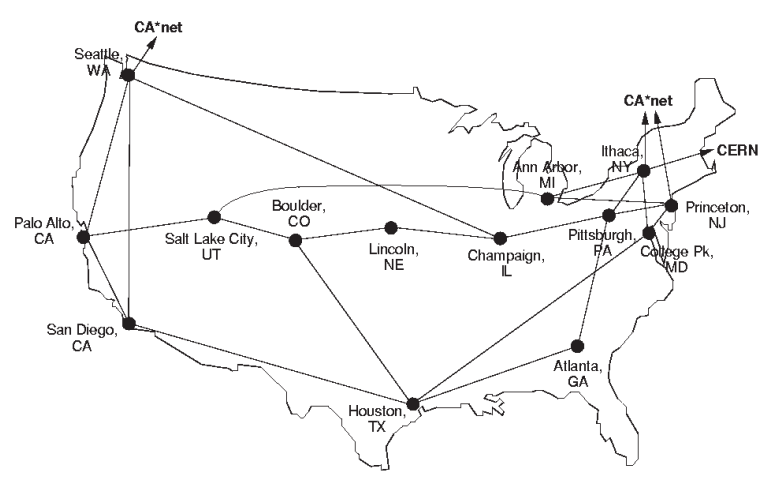

Figure 2. The NSFNet network.

Table IV. Objective functions and parameters assignment of eq. (1) used in simulations (trivial values)

\begin{tabular}{c|c}
\hline Objective function & $\begin{array}{c}\text { Parameters assignment } \\
\alpha_{G H G}-\alpha_{P W R}-\alpha_{L B}\end{array}$ \\
\hline \hline MinGHG & $1-0-0$ \\
\hline MinPower & $0-1-0$ \\
\hline LB & $0-0-1$ \\
\hline SP & all links's weight $=1$ \\
\hline
\end{tabular}

congestion in the network occurs (no blocking), in order to better appreciate their effect in terms of optimization without been affected by the trade-off introduced by the other ones (which will be discussed later). Therefore, the per-link cost function $c_{u v}(b)$ of eq. (1) has been initially set up to model the limit cases, according to the Table IV, where MinGHG, MinPower and LB objective functions model, respectively, the optimization of the GHG emissions, power consumption and the load balancing within the whole network. The unconstrained shortest path algorithm (SP) is also reported in the results for comparison.

In this way, the single objective functions (MinGHG, MinPower, LB and SP) are not affected by the other (sometime counter-productive) optimization objectives, and the upper-bounds to the achievable savings with the single optimization function can be precisely quantified.

In the following figures, the results in terms of power consumption, GHG emissions and average hop count (lightpath length) are reported for the MinGHG, MinPower, Load Balancing (LB) and Shortest Path (SP) algorithms. Results for the COST266 and NSFNet network topologies are organized from Fig. 2 to Fig. 6 according to the different connections lifetimes, respectively from 1 hour to 24 hours. 
In the frames (a) and (b) of Fig. 2 to Fig. 6, we can observe the power consumption achieved when the cost function is characterized by the four different individual objectives. The MinPower, thanks to its cost function based exclusively on the power scaling factors of the networks nodes, actually minimizes the power consumption of both networks in all experiments, keeping a larger gap with the other optimization schemes in COST266 than in NSFNet which, since having fewer nodes than the European network, requires in average shorter paths.

Among the remaining objective functions, which are all power-unaware, SP is not surprisingly the one that performs better; the selection of the shortest path has, in fact, a positive effect on the energy consumption, which is an additive metric (i.e., each network element of the path adds its non-null energy consumption), since the minimum number of intermediate devices are involved in each path selection. In the COST266 network, the trend of the power consumption of the SP algorithm is more regular than the one in NSFNet, which is due to the lower average connection degree of the NSFNet nodes.

The power consumption of the LB scheme is higher than the SP one, but it is on average lower than that of MinGHG. This is due to the fact that MinGHG will possibly determine longer paths than LB, in an effort to select always the lowest emitting nodes, passing through a higher number of (green) nodes to reach the destination, and statistically consuming more energy. The power consumption of MinGHG is therefore due to the particular path selection performed in order to minimize the emissions. Nontheless, a slight decrease in the power consumption of MinGHG, more evident in NSFNet (which, being less connected, provides more room for optimization), can be observed when the energy sources change interval increases, common to all the considered connection lifetimes. This phenomenon is due to the fact that the change in the energy sources acts as an indirect load balancing factor for the path selection criteria of MinGHG. Distributing the green sources randomly on the network elements, makes the MinGHG selection of the network nodes more balanced in order to follow the greenest nodes, statistically spreading the load uniformly over the whole network. In confirmation of such beneficial effect, we can observe that the decrease in the power consumption of MinGHG is more evident when the connections lifetime is shorter.
Since the power consumption of the network nodes does not change in time (i.e., the nodes consume the same amount of W/Gbps depending on their energy efficiency), MinPower shows a regular optimal trend as for the power consumption. The energy sources, instead, varying during the experiment once every $1,3,6,12$, or 24 hours, affect the path selection of the MinGHG scheme which is aware of the energy sources currently powering the devices. This awareness is reflected in the irregular trend shown for the GHG emissions achieved by MinGHG, reported in Fig. 2 to Fig. 6, frames (c) and (d). We can observe that, when the energy sources change slower than the connection lifetimes (e.g., connections lifetime $=1$ hour and energy sources change interval $=3$ hours), MinGHG obtains better results in terms of emissions with respect to SP algorithm, and outperforms even MinPower when the source change interval is one step longer (e.g., 6 or more hours for connections lifetime of 1 hour). In other words, even if MinGHG consume more energy than MinPower, this power, coming from greener sources, emits less GHG. This particular trend of MinGHG can be observed in all the experiments, and is due to longer lasting optimality of the MinGHG path selection criteria when the energy sources keep their value during all the connections lifetime. On the other hand, if the energy sources change interval is comparable to the connections lifetime, the path selection made at the connection setup time would soon become sub-optimal, and the connection that was "green" at the setup time, becomes "dirty" (GHG-emitting) during its lifetime. In general, when the connections lifetime is short enough compared to the energy sources change interval, MinGHG achieve the lowest carbon footprint with respect to the other algorithms, and such a trade-off is more evident when the gap among the time intervals increases. This observation suggests that high quality solutions can be found by appropriately weighting the $\alpha_{G H G}$ and $\alpha_{P W R}$ to obtain low emitting and low consuming lightpaths. The analysis of such a trade-off is discussed later in Sec. 6.1.

As expected, looking at the average path length reported in Fig. 2 to Fig. 6, frames (e) and (f), the SP algorithm exhibits the lowest hop count, followed by MinPower which, in an effort to minimize the (additive) power of the traversed nodes, minimizes also the average number of nodes (hops) per path, with some gap with SP due to the heterogeneous power scaling factors $\left(\varepsilon_{v}\right)$ assigned to the networks nodes. MinGHG has a regular trend, 
Table V. Objective functions and parameters assignment of eq. (1) used in simulations (trade-off study)

\begin{tabular}{c|c}
\hline Objective function & $\begin{array}{c}\text { Parameters assignment } \\
\alpha_{G H G}-\alpha_{P W R}-\alpha_{L B}\end{array}$ \\
\hline \hline Eco-friendly & $0,5-0,5-0$ \\
\hline Uniform & $0,333-0,333-0,333$ \\
\hline GHG+LB & $0,5-0-0,5$ \\
\hline Power+LB & $0-0,5-0,5$ \\
\hline
\end{tabular}

bearing slightly better trend than LB, that is somehow more irregular due to its load balancing criteria which tends to select different paths according to the already established ones in order to keep the network well balanced. Selecting the most balanced paths, at the beginning of the connections' routing process, will increase the cost of such paths, and other more peripheral paths will be eventually selected to route the incoming connections. As soon as these new paths are being selected more and more, their cost will increase and eventually exceed the cost of the paths that were selected first, making these ones again more convenient for routing the remaining connections, and so on, causing the observed pseudosinusoidal behavior of the LB, which can be observed also, with smaller magnitude, in the GHG emissions and power consumption results.

After assessing the effectiveness of the proposed scheme against the individual component optimization goals, we study the effects of the trade-off between the different objectives by letting the three $\alpha_{x}$ parameters vary within a specified range of combined values covering most of the possible scenarios.

\subsection{Tuning the $\alpha_{x}$ parameters}

The scalarization technique employed in the mathematical model allows to select arbitrary values of the $\alpha_{x}$ parameters, provided that $\sum_{x} \alpha_{x}=1$. Nonetheless, from the network optimization point of view, the optimal selection of these parameters does not exist in general, but it depends on the particular operational objective of the network operator. Therefore, considering that the results for the extreme (trivial) values of $\alpha_{x}$ have been already presented, a selection of four notable combination of $\alpha_{x}$ covering most intermediate dynamics are here studied (see Tab. V).

In particular, we consider an Eco-friendly optimization objective, in which GHG emissions and power consumption are equally balanced; a Uniform objective function in which the $\alpha_{x}$ weights are equally distributed; and finally, the GHG+LB and Power+LB objective functions which assign one half of the weight to LB and the other half to GHG and Power, respectively, covering in this way all the possible combinations of two objective functions at a time.

Since for the different connections lifetimes we obtained similar results, we present only the intermediate cases, i.e. for connections lifetimes of 3 and 6 hours, reported in Fig. 8 and Fig. 9 respectively, for both the COST266 and NSFNet networks.

The first observation is that, by tuning the $\alpha_{x}$ weights by assigning intermediate values, we obtain results whose scale (i.e., the $Y$ axis) is contained in the interval of the previous trivial cases, as expected, since such extreme cases represent the upper-bounds to the achievable savings with a single optimization function. In other words, by properly tuning the $\alpha_{x}$ parameters, we obtain smoother results that pursue multi-objective optimization functions. This situation is particularly evident for the Eco-friendly approach, which decreases both the power consumption of MinGHG and the GHG emissions of MinPower, the latter starting from connections lifetime equal or lower than the energy sources change interval.

As for the the power consumption, reported in Fig. 8 and Fig. 9 frames (a) and (b), we observe that GHG+LB is the more emitting strategy, since it do not take into account the power consumption of the involved network elements but tries to route the connections on the greenest, best-balanced paths. As expected, Power+LB is the lowest emitting strategy, since it is the one with the greatest weight assigned to the power consumption $\left(\alpha_{\text {Power }}=\right.$ 0,5) together with the Eco-friendly, which however shows the effects of the longer paths chosen by the GHG optimization sub-criteria $\left(\alpha_{G H G}=0,5\right)$. Uniform distribution of weights provides the best-balanced results in all tests, making it attractive for conservative network operation while not discarding eco-concerns.

Taking a look at the GHG emissions of the four strategies, we can observe a common behavior of the GHG-aware functions (Eco-friendly, GHG+LB and Uniform), which tend to lower their emissions as the energy sources change interval increases. The only function that presents a more regular trend is the Power+LB which is unaware of the emissions. The threshold, or cut-point, between Power+LB and the other GHG-aware functions is located at the connections 


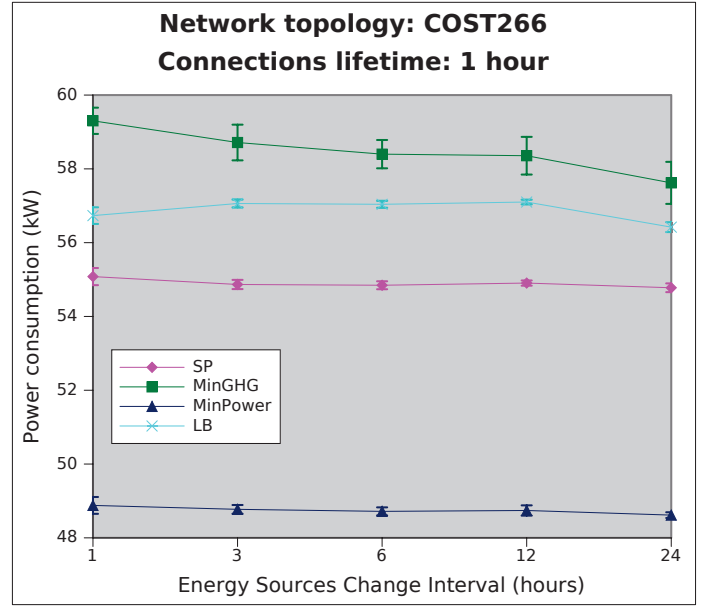

(a) Cost 266, Power Consumption

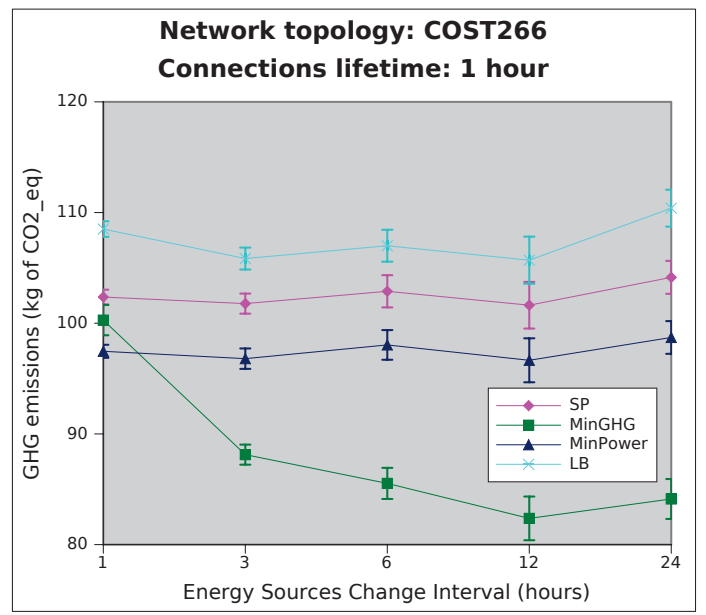

(c) Cost 266, GHG Emissions

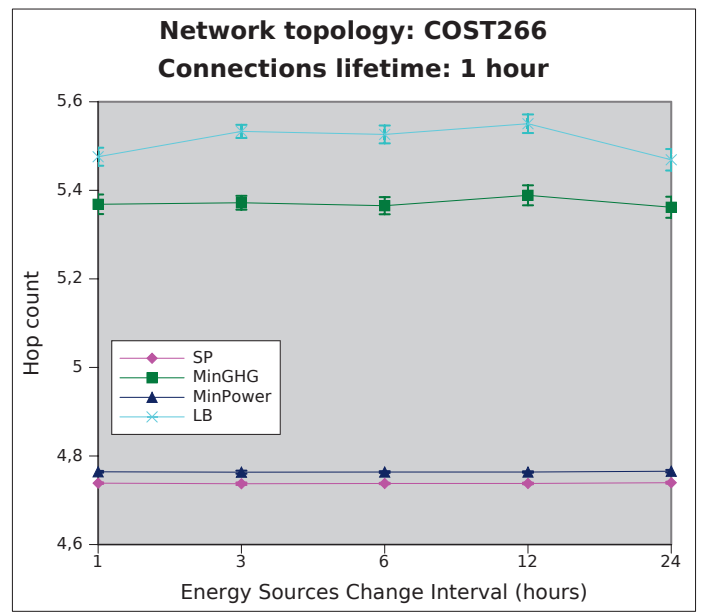

(e) Cost 266, Hop Count

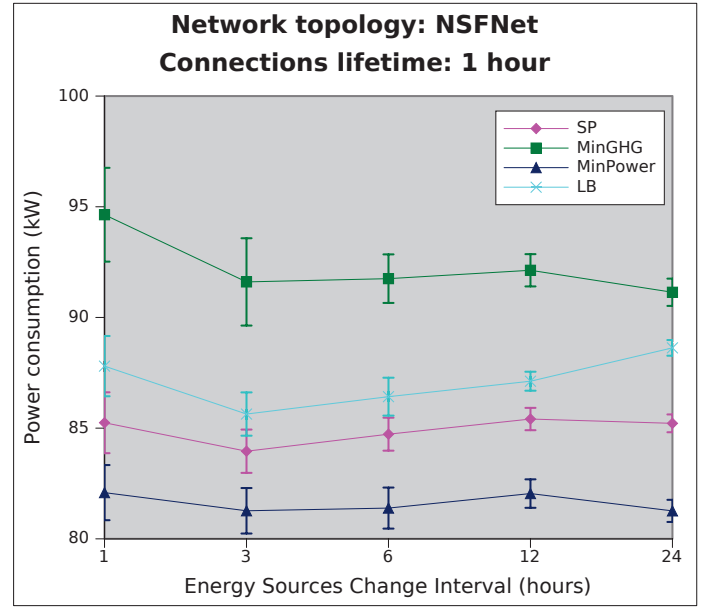

(b) NSFNet, Power Consumption

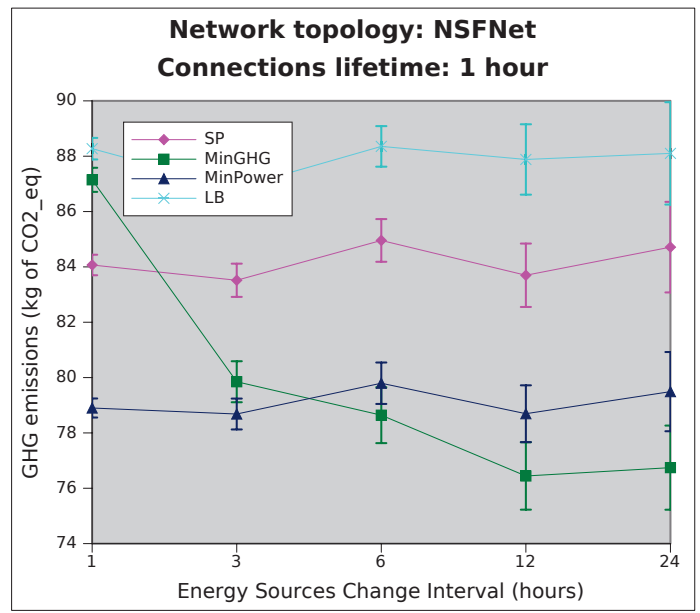

(d) NSFNet, GHG Emissions

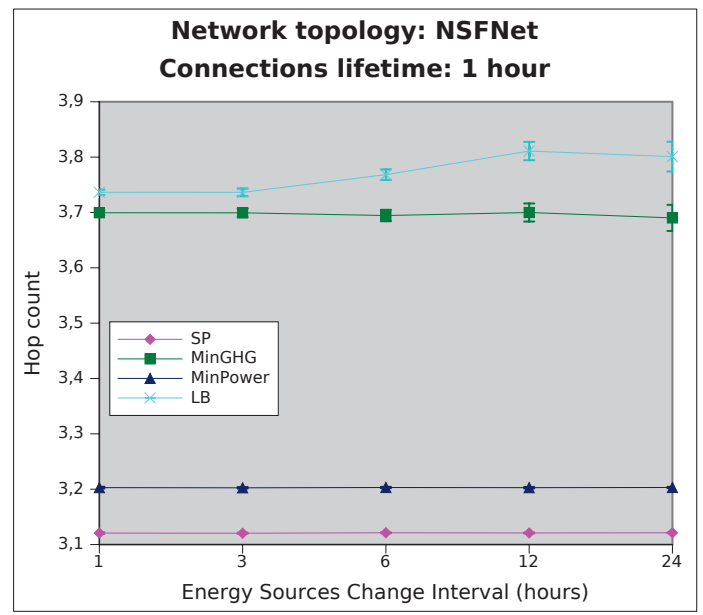

(f) NSFNet, Hop Count

Figure 3. Simulation results for the case study topologies with connection lifetime $1 \mathrm{~h}$. 


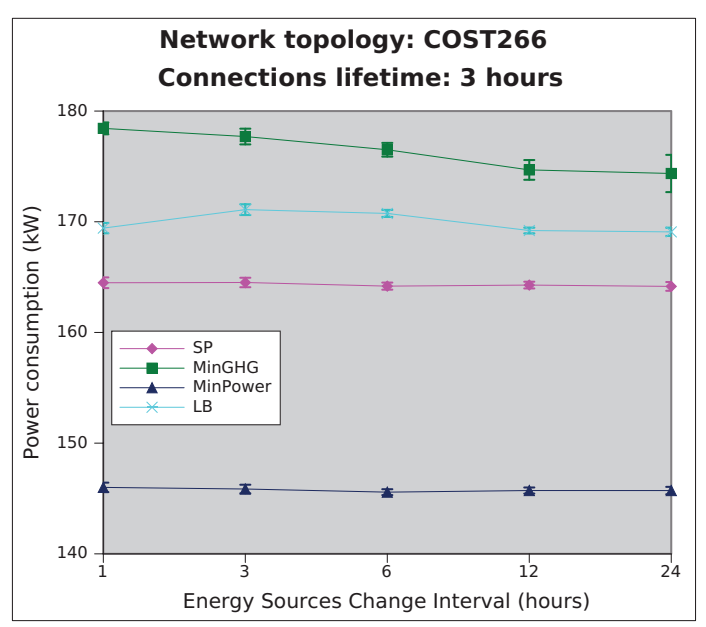

(a) Cost 266, Power Consumption

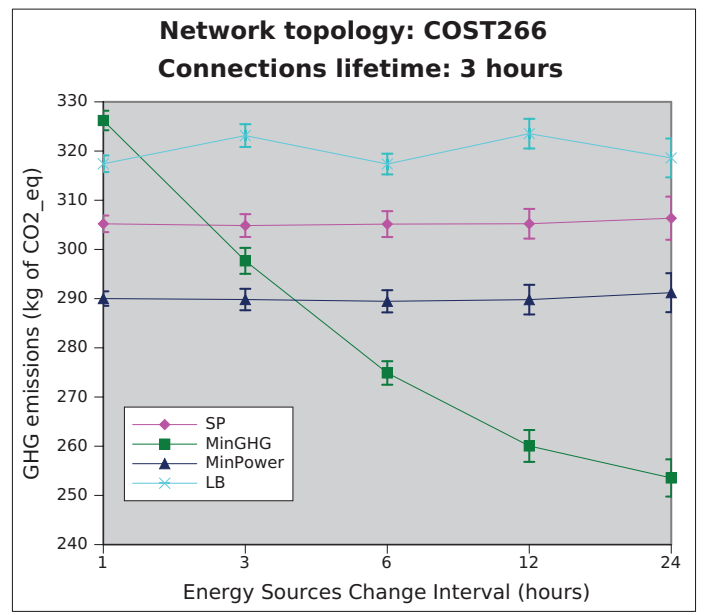

(c) Cost 266, GHG Emissions

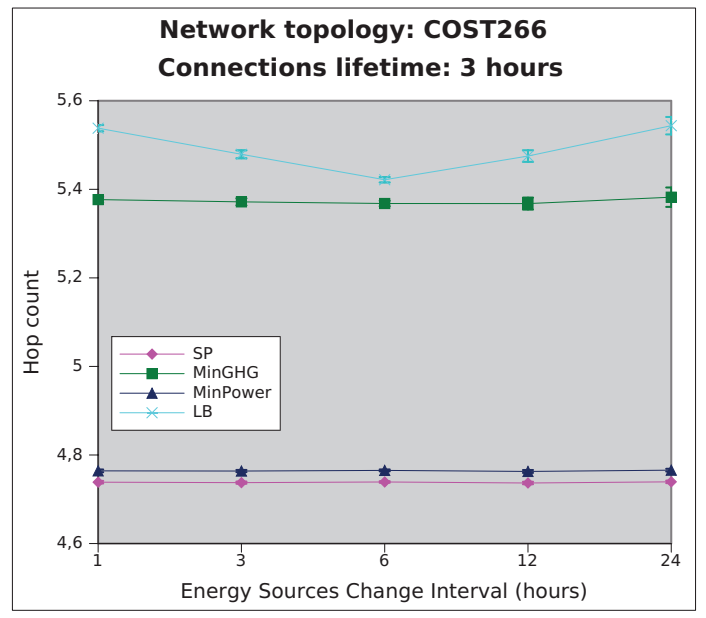

(e) Cost 266, Hop Count

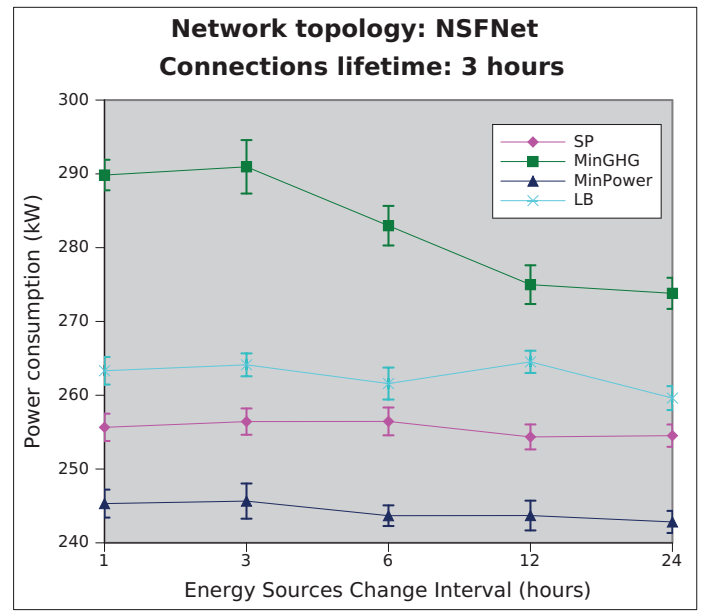

(b) NSFNet, Power Consumption

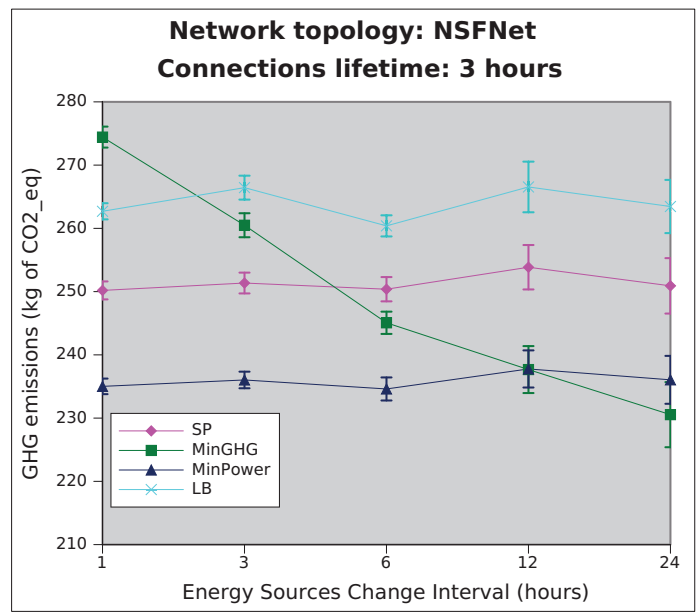

(d) NSFNet, GHG Emissions

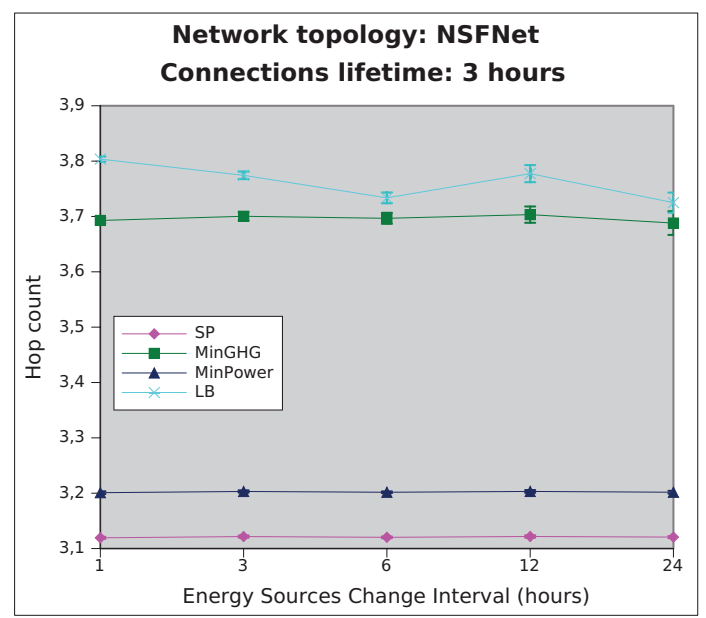

(f) NSFNet, Hop Count

Figure 4. Simulation results for the case study topologies with connection lifetime $3 \mathrm{~h}$. 


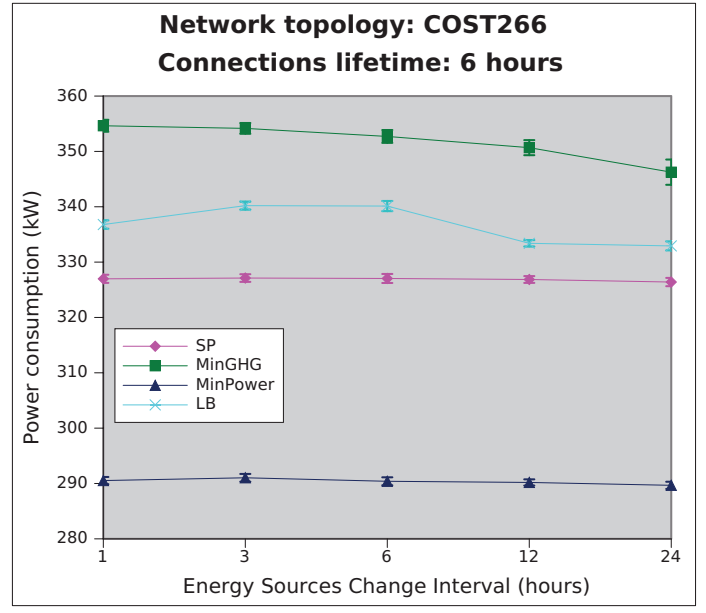

(a) Cost 266, Power Consumption

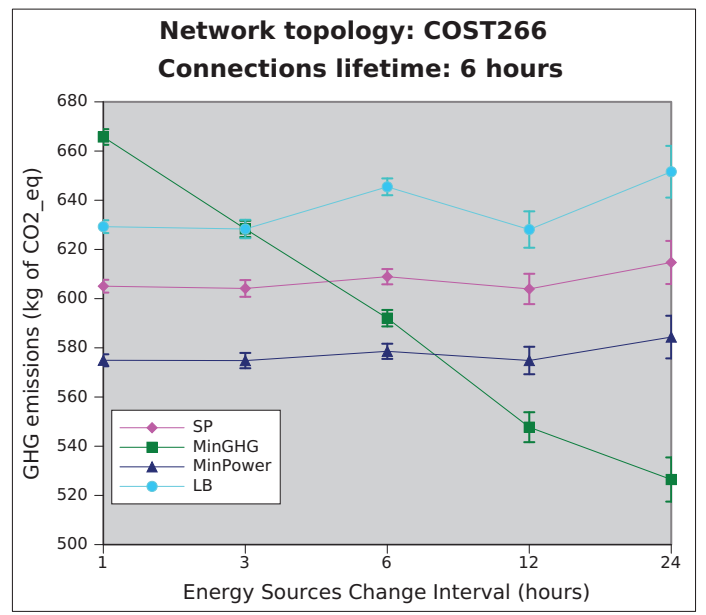

(c) Cost 266, GHG Emissions

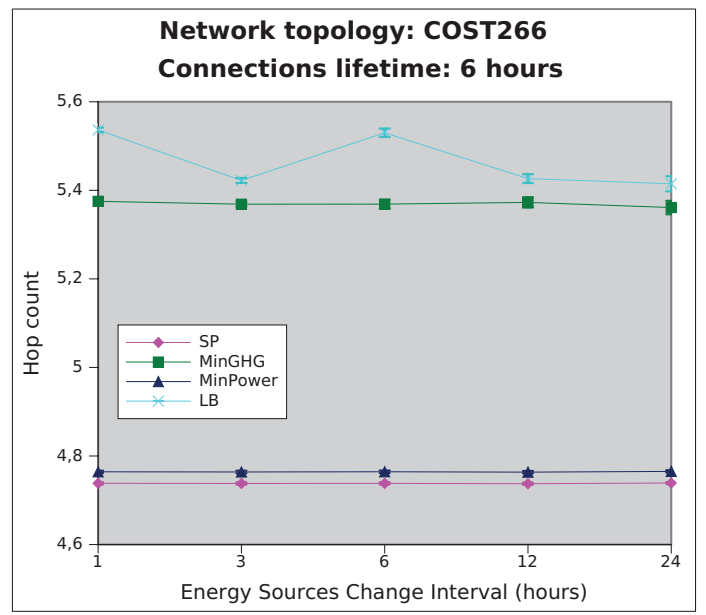

(e) Cost 266, Hop Count

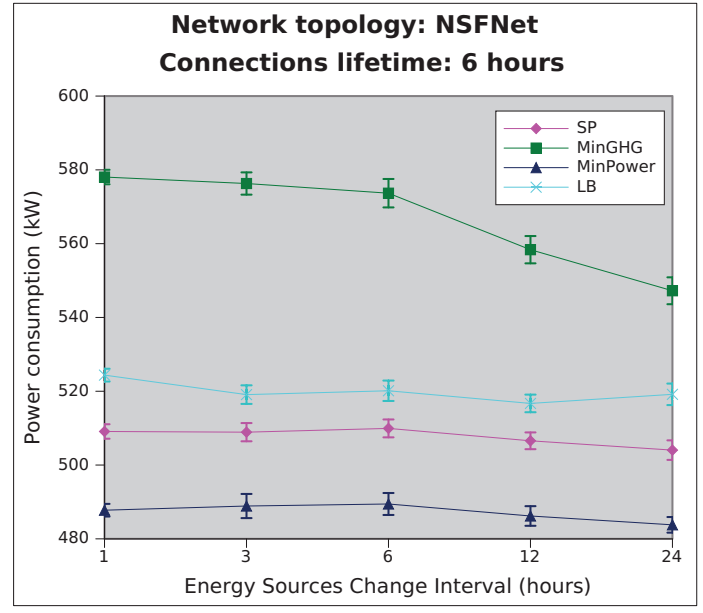

(b) NSFNet, Power Consumption

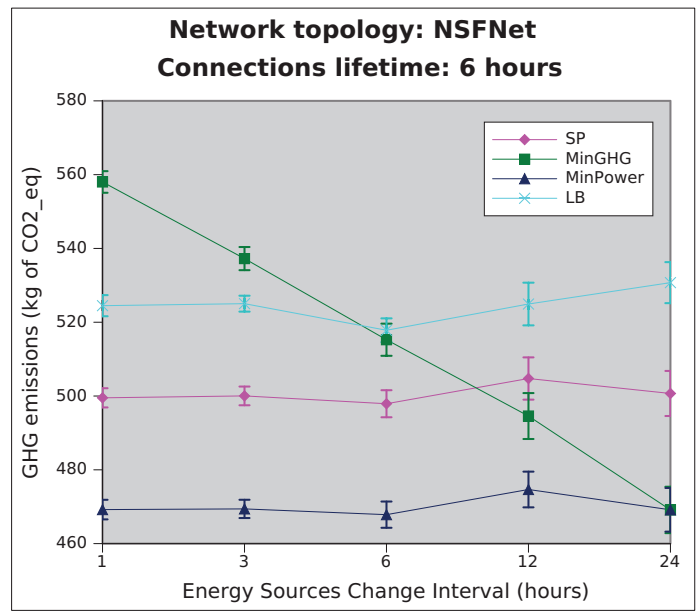

(d) NSFNet, GHG Emissions

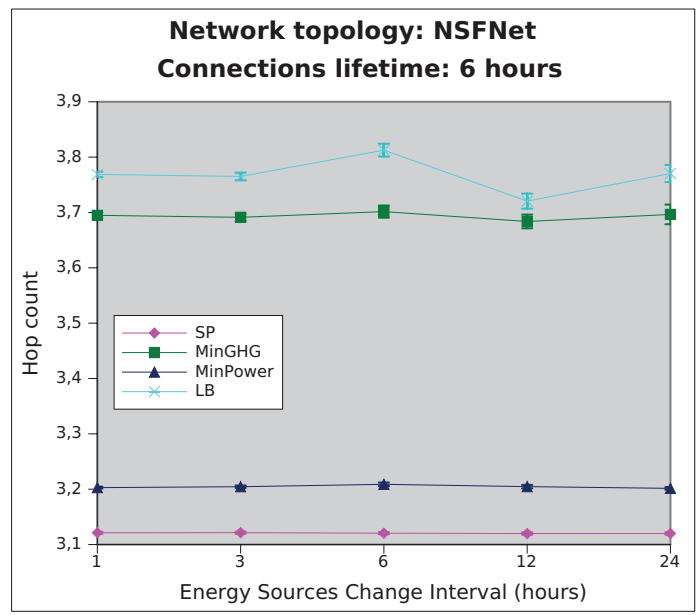

(f) NSFNet, Hop Count

Figure 5. Simulation results for the case study topologies with connection lifetime $6 \mathrm{~h}$. 


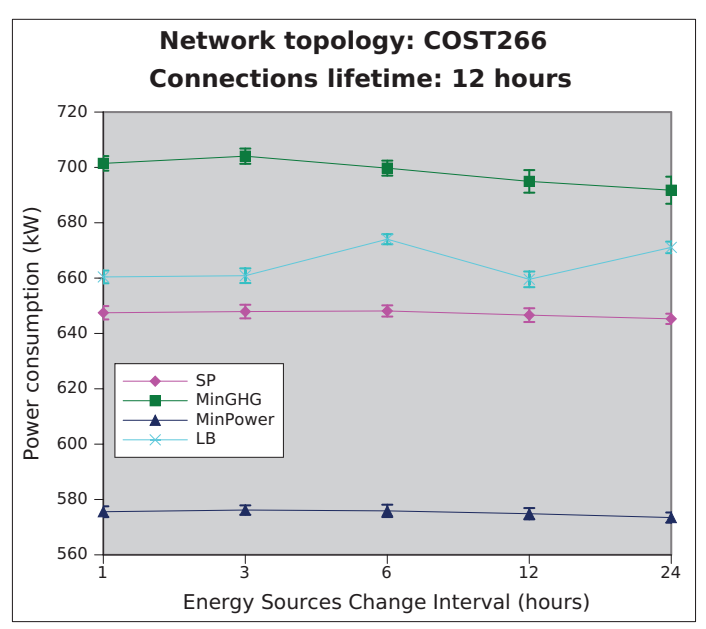

(a) Cost 266, Power Consumption

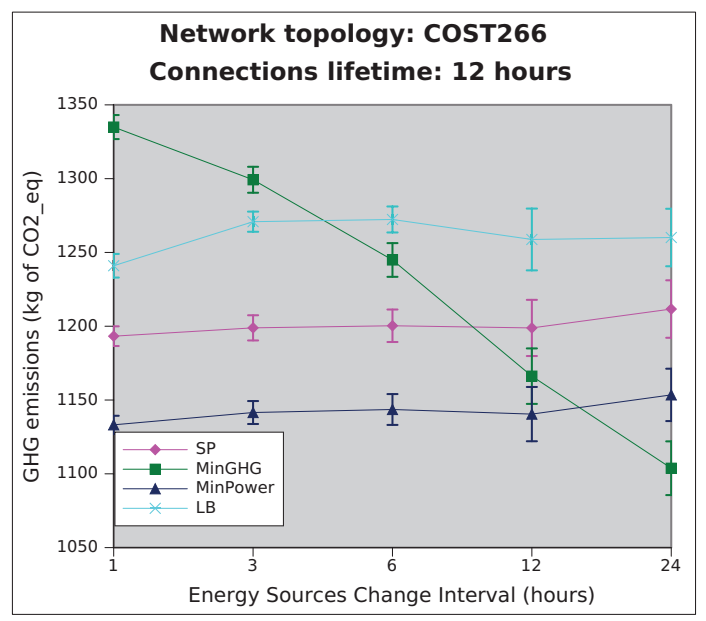

(c) Cost 266, GHG Emissions

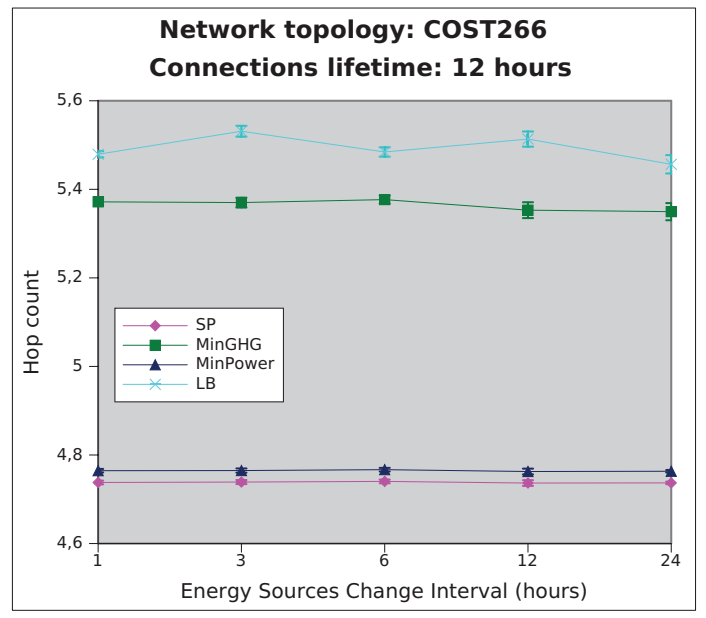

(e) Cost 266, Hop Count

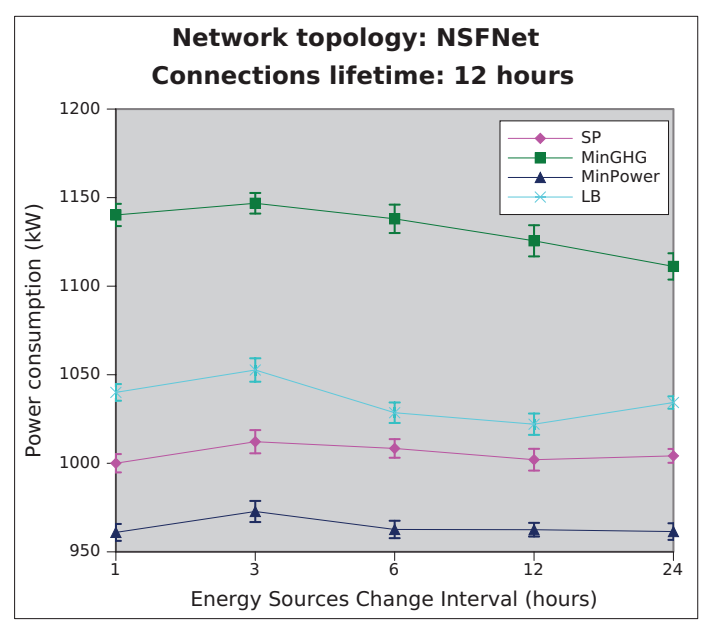

(b) NSFNet, Power Consumption

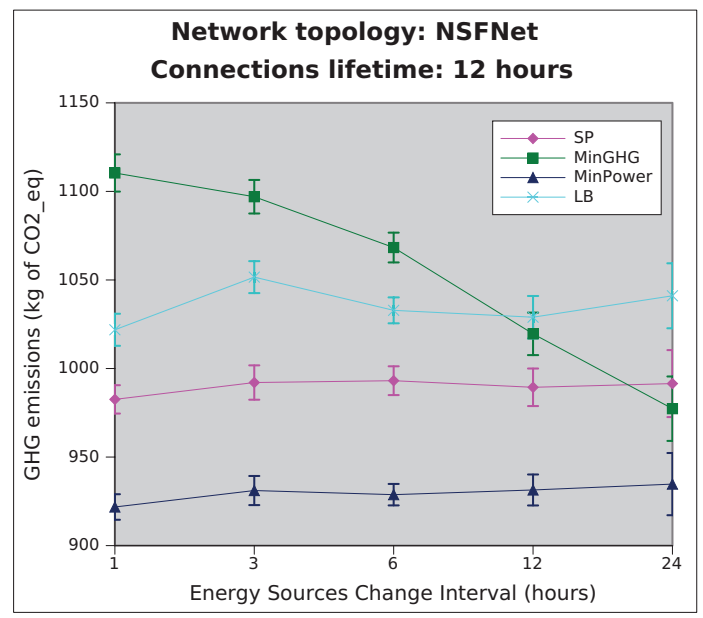

(d) NSFNet, GHG Emissions

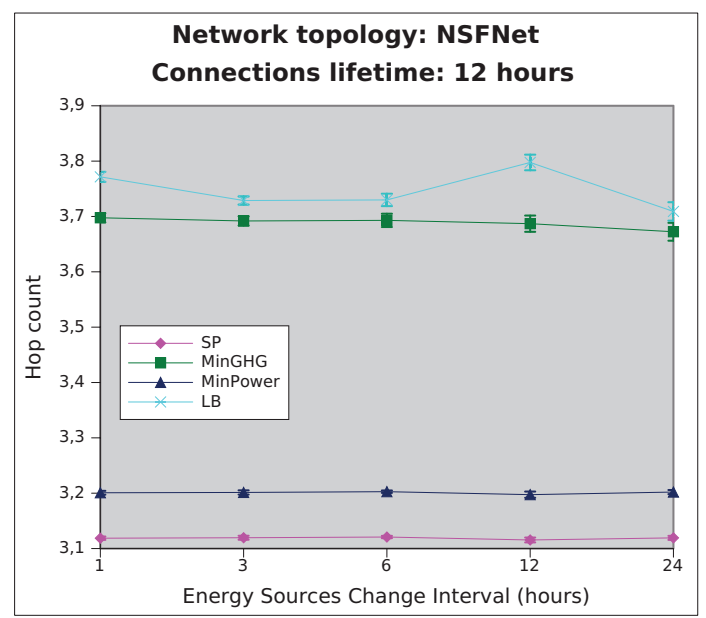

(f) NSFNet, Hop Count

Figure 6. Simulation results for the case study topologies with connection lifetime $12 \mathrm{~h}$. 


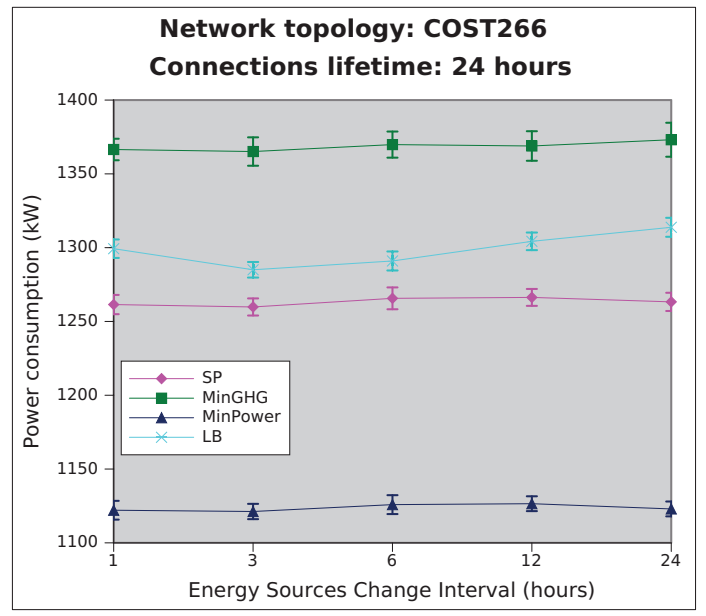

(a) Cost 266, Power Consumption

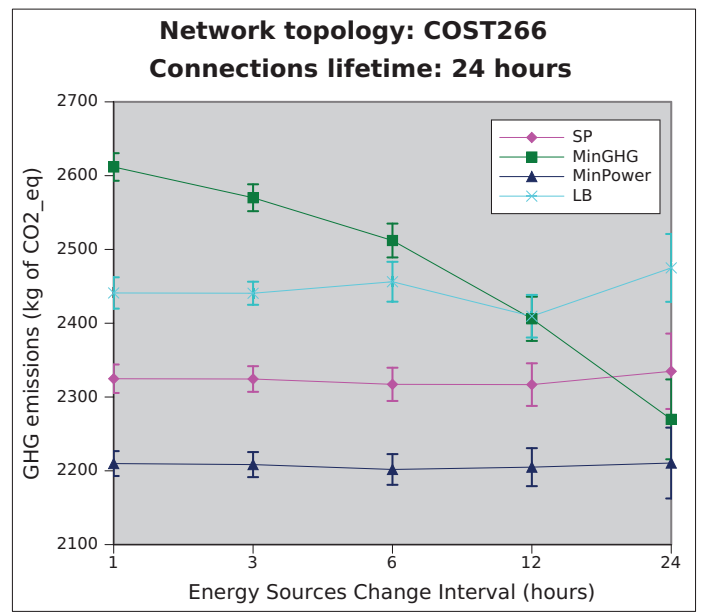

(c) Cost 266, GHG Emissions

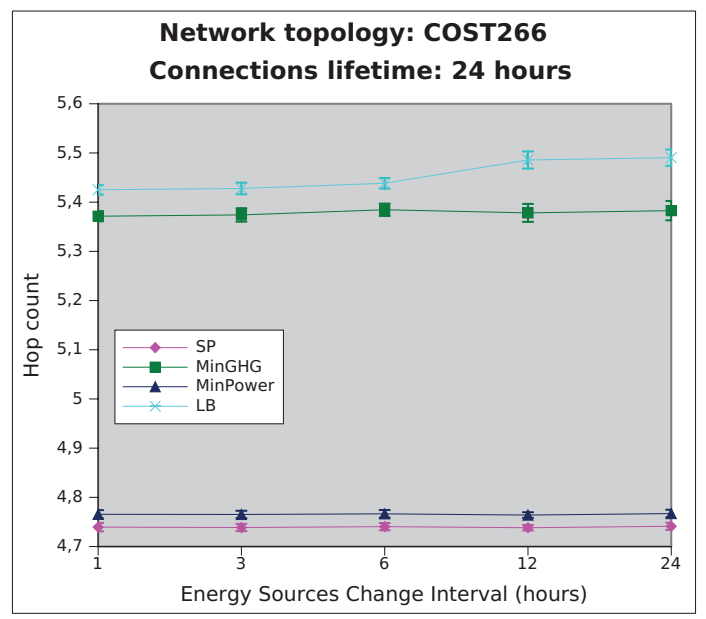

(e) Cost 266, Hop Count

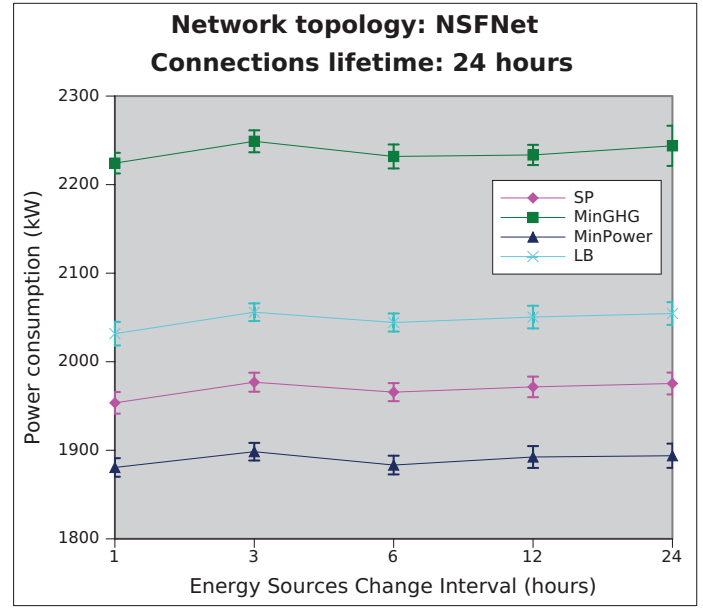

(b) NSFNet, Power Consumption

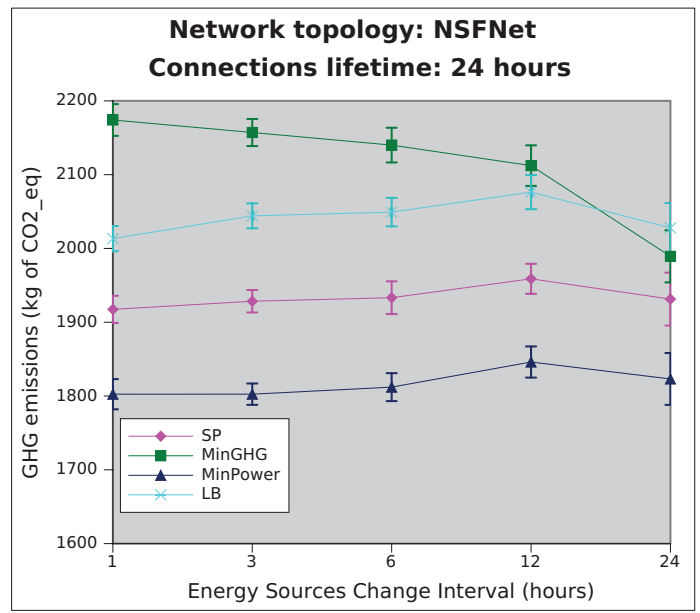

(d) NSFNet, GHG Emissions

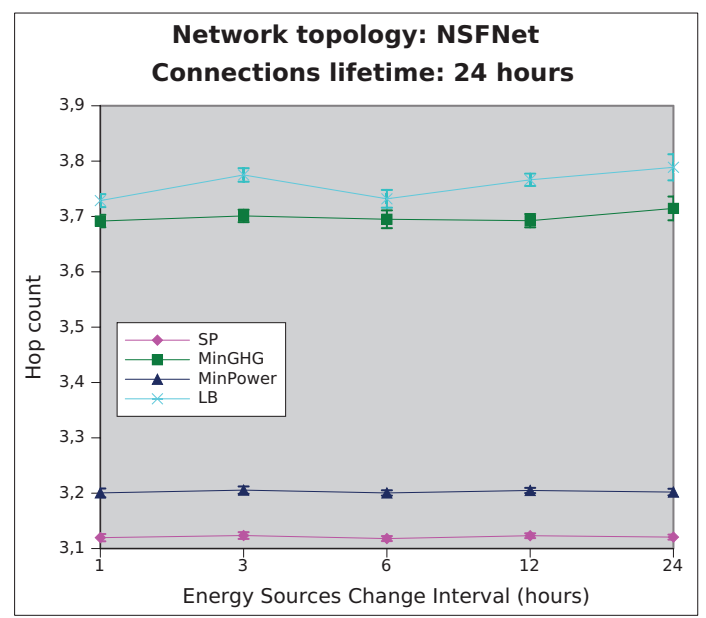

(f) NSFNet, Hop Count

Figure 7. Simulation results for the case study topologies with connection lifetime $24 \mathrm{~h}$. 


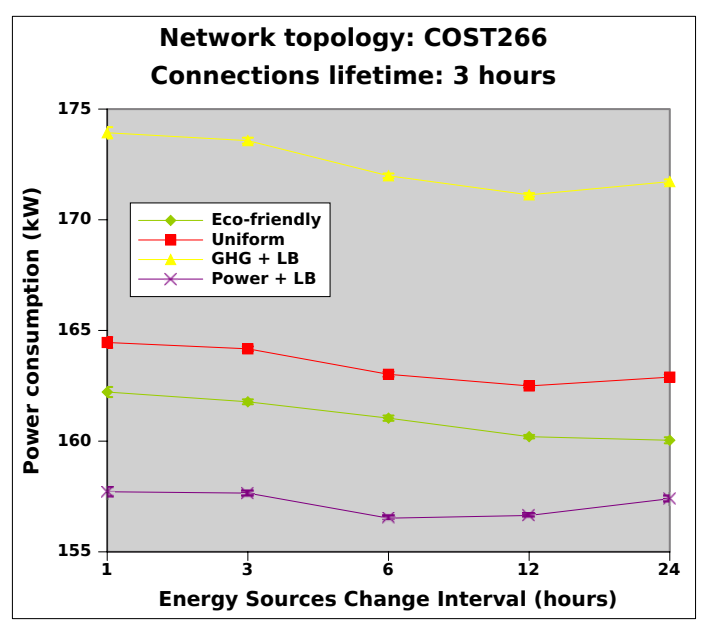

(a) Cost 266, Power Consumption

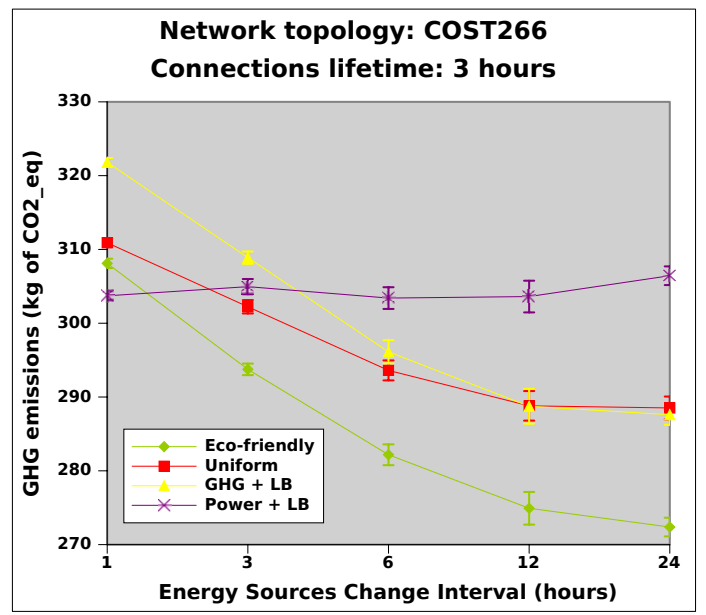

(c) Cost 266, GHG Emissions

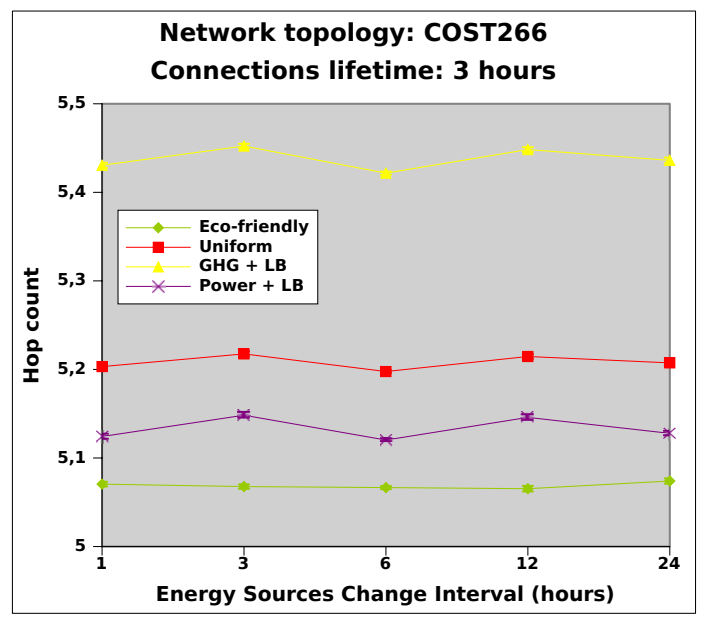

(e) Cost 266, Hop Count

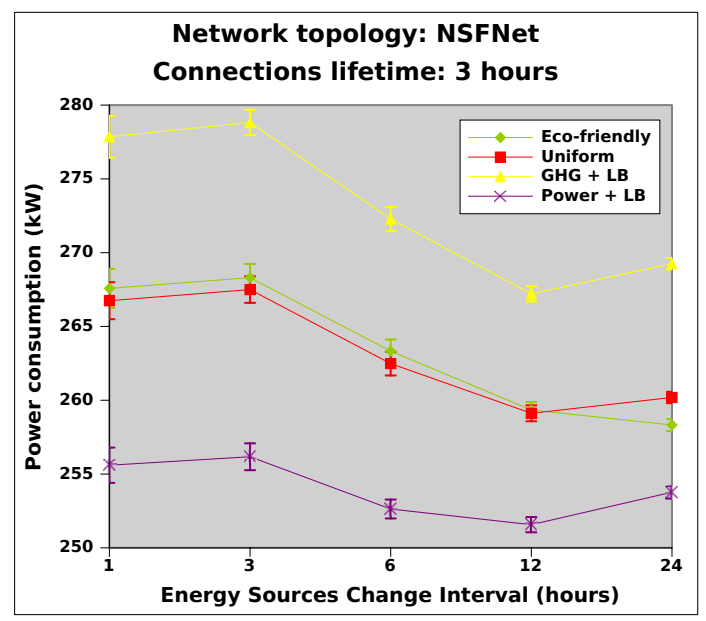

(b) NSFNet, Power Consumption

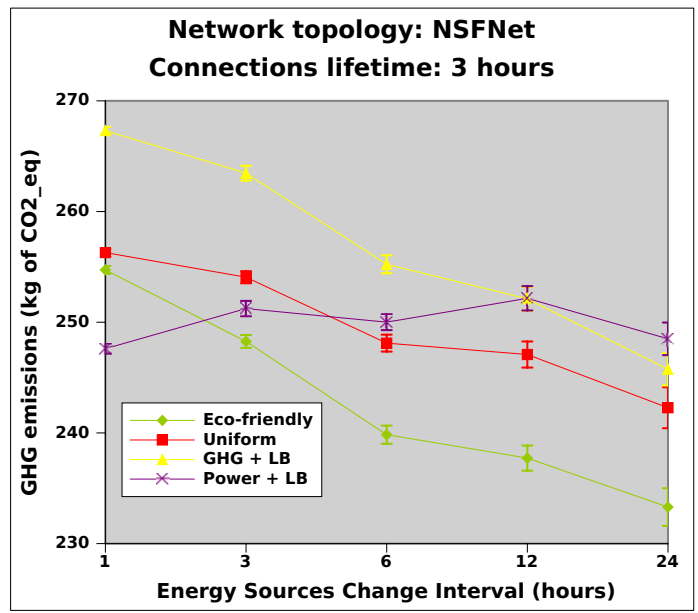

(d) NSFNet, GHG Emissions

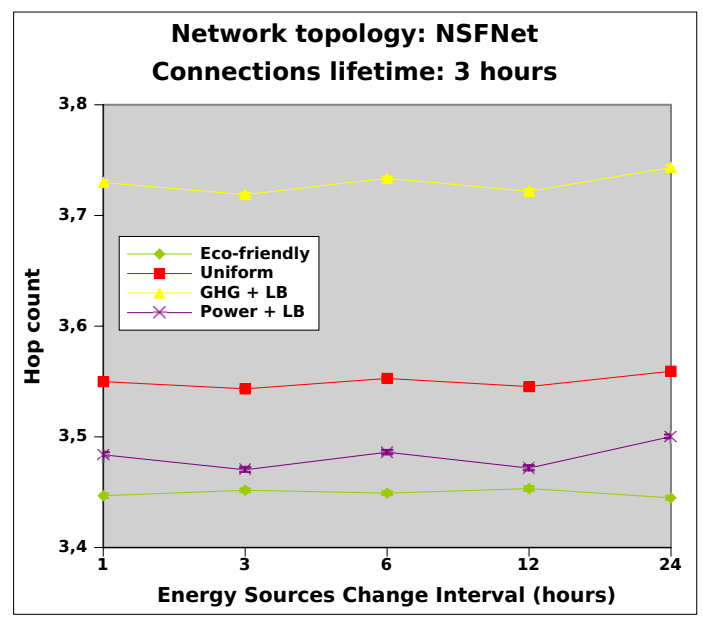

(f) NSFNet, Hop Count

Figure 8. Simulation results for the trade-off case study topologies with connection lifetime $3 \mathrm{~h}$. 


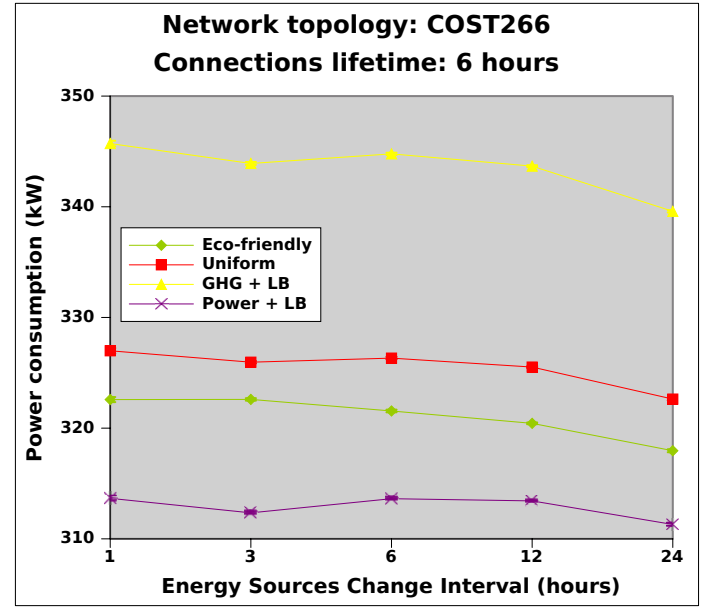

(a) Cost 266, Power Consumption

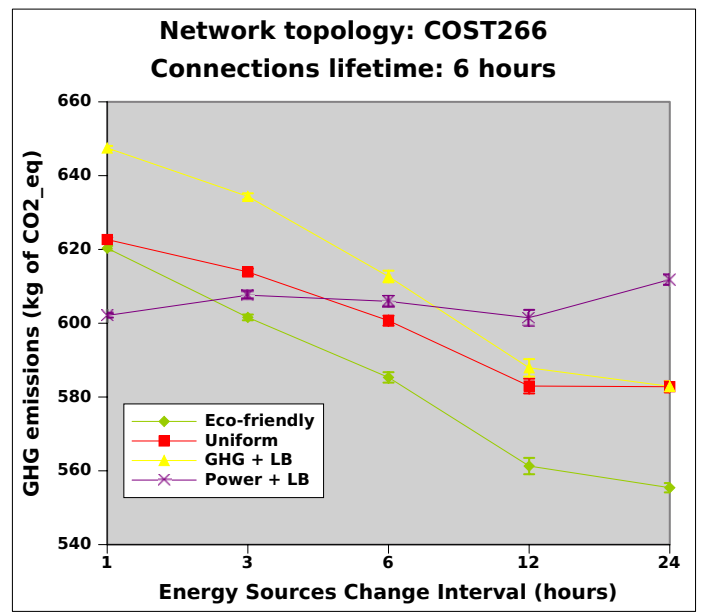

(c) Cost 266, GHG Emissions

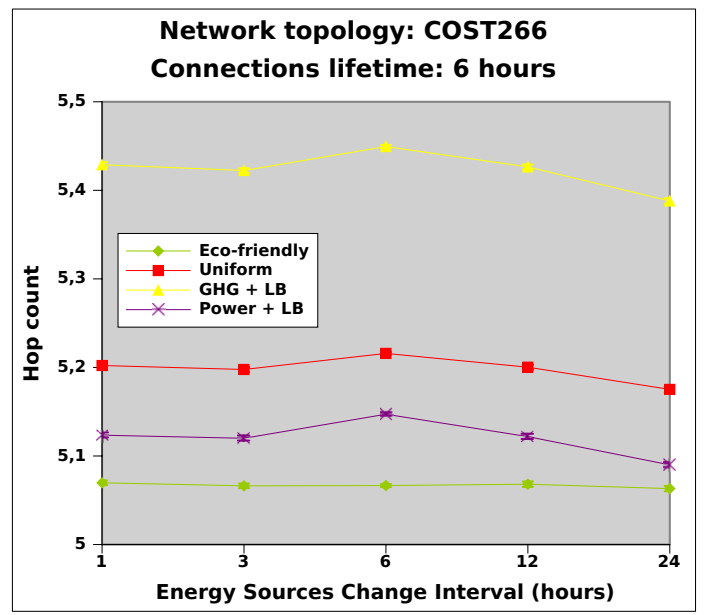

(e) Cost 266, Hop Count

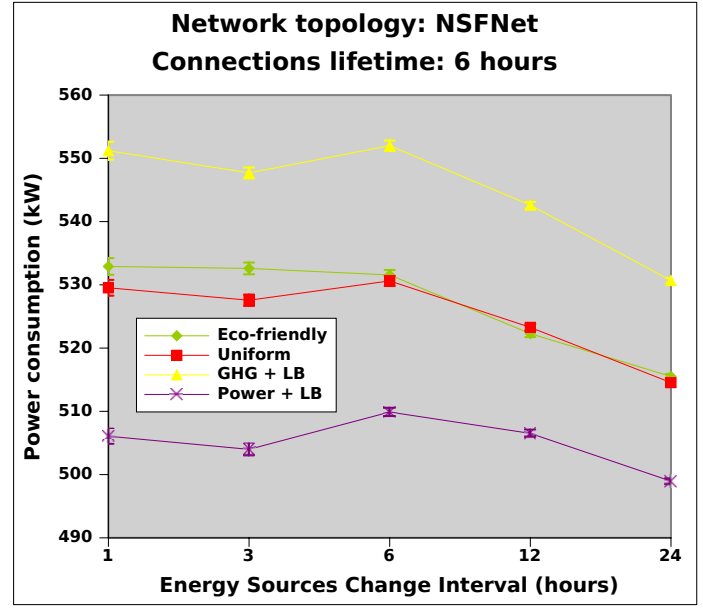

(b) NSFNet, Power Consumption

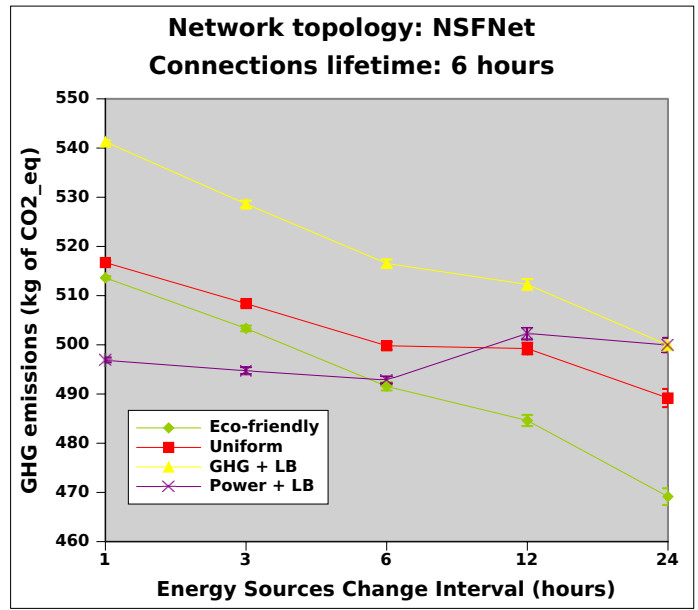

(d) NSFNet, GHG Emissions

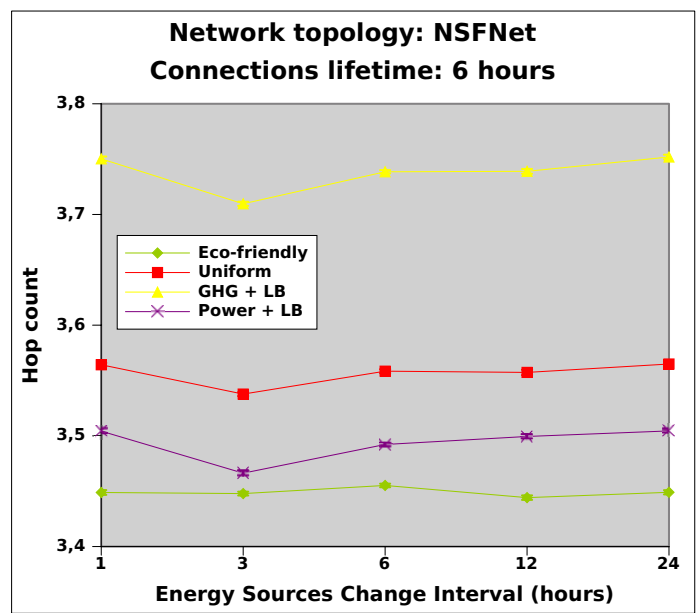

(f) NSFNet, Hop Count

Figure 9. Simulation results for the trade-off case study topologies with connection lifetime $6 \mathrm{~h}$ 
lifetime, which marks the threshold beyond which the GHG-aware functions are profitable.

The higher power consumption of GHG+LB is confirmed by looking at the average hop count graphics, which actually reveals that GHG+LB lightpaths are sensibly longer than the other strategies' ones. The shortest paths are chosen by the Eco-friendly function, as a consequence of the double minimization objectives, minimizing power and GHG emissions, followed by Power+LB. As expected, the Uniform function obtains average-length paths, being a balanced trade-off among the three different optimization objectives.

\section{CONCLUSIONS}

Traditional RWA schemes are showing their limitations when coping with the emerging problem of the information and communication society, in which the sustainability plays an increasingly important role, for both reducing the network's operational expenditures and environmental impacts. Towards this goal, a new RWA scheme has been presented which, by extending the traditional OSPFTE routing protocol, considers as additional network engineering parameters the power consumption and GHG emissions of the network elements, in order to minimize the energy consumption of the network and its carbon footprint. Two different optimization functions have been presented, namely MinPower and MinGHG, with the objective of minimizing the power consumption and the GHG emissions respectively. An extensive simulation study has been performed on the European COST266 and the US NSFNet network topologies, and the simulation results have been carefully analyzed, highlighting the benefits and the drawbacks of the presented algorithms and optimization objectives, and comparing them also with a well-known reference algorithm, used in almost all the available link state routing implementations. Results show that MinPower minimizes the energy consumption, while keeping very limited the GHG emissions and making an efficient use of the network resources, evident for the low average path length. MinGHG, instead, due to the variability of the energy sources, achieves the lowest emissions only under favorable conditions, i.e. when the connection lifetime is short enough compared with the energy sources change interval. In general, if limited or no information is known on the energy sources, the best conservative choice is to employ a power minimization algorithm, such as MinPower, and using MinGHG only if a carbon containment strategy is put in place and the network operators pays according to the quantity of GHG emissions. Last, an analysis of the trade-off among the different optimization objective has been studied, showing that a good balance between the minimization of GHG emissions and of power consumption can be achieved when pursuing Eco-friendly optimization objective, while the Uniform distribution of importance among GHG, Power and LB represents an attractive solution in most general cases, in which the traditional network objectives are considered together with the emerging, eco-aware concerns of GHG emissions and power consumption minimization.

\section{ACKNOWLEDGEMENTS}

This work was supported in part by the Spanish Ministry of Science and Innovation under the DOMINO project (TEC2010-18522) and the Catalan Government under the contract SGR 1140. The authors also acknowledge the support received from OPNET Technologies Inc.

\section{REFERENCES}

1. St Arnaud B. Ict and global warming: Opportunities for innovation and economic growth. http://docs.google.com/Doc? id=dgbgjret $\backslash 2767 \mathrm{dxpbdvcf}$.

2. Bt announces major wind power plans.

http://www.btplc.com/News/

Articles/Showarticle.cfm?ArticleID= dd615e9c-71ad-4daa-951a-55651baae5bb 2007.

3. Pileri S. Energy and Communication: engine of the human progress. INTELEC 2007, Rome, Italy, Sept. 2007.

4. Souchon Foll L. TIC et Énergétique: Techniques d'estimation de consommation sur la hauteur, la structure et l'évolution de l'impact des TIC en France. Ph.D. dissertation, Orange Labs/Institut National des Télécommunications, 2009. 
5. Baliga J, Ayre R, Hinton K, Sorin WV, Tucker RS. Energy consumption in optical ip networks. Journal of Lightwave Technology 2009; 27(13):2391-2403.

6. Ricciardi S, Santos-Boada G, Klinkowski M, Careglio D, Palmieri F. Towards Service Orchestration between Smart Grids and Telecom Networks. Energy Efficiency in Large Scale Distributed Systems (EE-LSDS 2013), Viena, Austria, Apr. 2013.

7. Gupta M, Singh S. Greening of the internet. Proceedings of the 2003 conference on Applications, technologies, architectures, and protocols for computer communications, SIGCOMM '03, ACM: New York, NY, USA, 2003; 19-26.

8. Al-Kanj L, Dawy Z. Impact of network parameters on the design of energy-aware cooperative content distribution protocols. Transactions on Emerging Telecommunications Technologies 2013; 24(3):317-330, doi: 10.1002/ett.2552. URL http://dx.doi.org/ $10.1002 /$ ett.2552.

9. Zhang X, Zhang J, Huang Y, Wang W. On the study of fundamental trade-offs between qoe and energy efficiency in wireless networks. Transactions on Emerging Telecommunications Technologies 2013; 24(3):259-265, doi:10.1002/ett.2640. URL http: //dx.doi.org/10.1002/ett.2640.

10. Idzikowski F, Orlowski S, Raack C, Woesner H, Wolisz A. Saving energy in IP-over-WDM networks by switching off line cards in low-demand scenarios. Optical Network Design and Modeling (ONDM), 2010 14th Conference on, 2010; 1 -6, doi:10.1109/ ONDM.2010.5431569.

11. Wu Y, Chiaraviglio L, Mellia M, Neri F. Poweraware routing and wavelength assignment in optical networks. Optical Communication, 2009. ECOC'09. 35th European Conference on, IEEE, 2009; 1-2.

12. Ricciardi S, Careglio D, Fiore U, Palmieri F, SantosBoada G, Solé-Pareta J. Analyzing local strategies for energy-efficient networking. Networking Workshops, Lecture Notes in Computer Science, vol. 6827, 2011; 291-300.

13. Kist A, Aldraho A. Dynamic topologies for sustainable and energy efficient traffic routing. Computer Networks 2011; .

14. Arai D, Yoshihara K. Eco-friendly distributed routing protocol for reducing network energy consumption. Network and Service Management (CNSM), 2010
International Conference on, Oct.; 104-111, doi:10. 1109/CNSM.2010.5691325.

15. Warip M, Glesk I, Andonovic I. Gmpls energy efficiency scheme for green photonic networks. Transparent Optical Networks (ICTON), 2010 12th International Conference on, 27 2010-July 1; 1-4, doi:10.1109/ICTON.2010.5549034.

16. Dong X, El-Gorashi T, Elmirghani JMH. Low carbon emission ip over wdm network. Communications (ICC), 2011 IEEE International Conference on, June; 1-6, doi:10.1109/icc.2011.5962482.

17. Dong X, El-Gorashi T, Elmirghani J. Renewable energy for low carbon emission ip over wdm networks. Optical Network Design and Modeling (ONDM), 2011 15th International Conference on, Feb.; 1-6.

18. Ricciardi S, Careglio D, Palmieri F, Fiore U, Santos-Boada G, Solé-Pareta J. Energy-aware RWA for WDM networks with dual power sources. Communications (ICC), 2011 IEEE International Conference on, 2011; 1-6, doi:10.1109/icc.2011. 5962432.

19. Ricciardi S, Careglio D, Santos-Boada G, SoléPareta J, Fiore U, Palmieri F. Towards an energyaware internet: modeling a cross-layer optimization approach. Telecommunication Systems 2011; :122doi:10.1007/s11235-011-9645-7.

20. Boddie M, Schondienst T, Vokkarane V. Dual power source aware algorithms for green optical network survivability. Online Conference on Green Communications (GreenCom), 2012 IEEE, 2012; 3237, doi:10.1109/GreenCom.2012.6519612.

21. Gattulli M, Tornatore M, Fiandra R, Pattavina A. Low-carbon routing algorithms for cloud computing services in ip-over-wdm networks. Communications (ICC), 2012 IEEE International Conference on, IEEE, 2012; 2999-3003.

22. Ricciardi S, Palmieri F, Fiore U, Careglio D, Santos-Boada G, Solé-Pareta J. An energy-aware dynamic rwa framework for next-generation wavelength-routed networks. Computer Networks 2012; 56(10):2420-2442.

23. Fang W, Wang Z, LIoret J, Zhang D, Yang Z. Optimising data placement and traffic routing for energy saving in backbone networks. Transactions on Emerging Telecommunications Technologies 2013; 
:n/a-n/adoi:10.1002/ett.2774. URL http://dx . doi.org/10.1002/ett. 2774 .

24. Kompella K, Rekhter Y. Ospf extensions in support of generalized multi-protocol label switching (gmpls). Technical Report, IETF RFC 36302005.

25. Awduche D, Berger L, Gan D, Li T, Srinivasan V, Swallow G. Rsvp-te: Extensions to rsvp for lsp tunnels. Technical Report, IETF RFC 32091997.

26. Andersson L, Callon R, Dantu R, Wu L, Doolan P, Worster T, Feldman N, Fredette A, Girish M, Gray E, et al.. Constraint-based lsp setup using ldp. Technical Report, IETF RFC 32122002.

27. Bone project, wp 21 tp green optical networks, $\mathrm{d} 21.2 \mathrm{~b}$ report on $\mathrm{y} 1$ and updated plan for activities 2009. URL

http://www.ict-bone.eu/portal/

landing_pages/bone_deliverables/ bone_216863_-_d21-2b_-_report_y1.pdf.

28. Chabarek J, Sommers J, Barford P, Estan C, Tsiang

$\mathrm{D}$, Wright S. Power awareness in network design and routing. INFOCOM 2008. The 27th Conference on Computer Communications. IEEE, 2008; 457-465, doi:10.1109/INFOCOM.2008.93.

29. Ricciardi S, Careglio D, Palmieri F, Fiore U, SantosBoada G, Solé-Pareta J. Energy-oriented models for wdm networks. Broadband Communications, Networks, and Systems, Lecture Notes of the Institute for Computer Sciences, Social Informatics and Telecommunications Engineering, vol. 66. Springer Berlin Heidelberg, 2012; 534-548, doi:10.1007/ 978-3-642-30376-0 \39. URL http: / / dx. doi . org/10.1007/978-3-642-30376-0_39.

30. Van Heddeghem W, Idzikowski F, Vereecken W, Colle D, Pickavet M, Demeester P. Power consumption modeling in optical multilayer networks. Photonic Network Communications 2012; 24(2):86-102.

31. Kodialam M, Lakshman T. Minimum interference routing with applications to mpls traffic engineering. INFOCOM 2000. Nineteenth Annual Joint Conference of the IEEE Computer and Communications Societies. Proceedings. IEEE, vol. 2, IEEE, 2000; 884-893.

32. Caramia M, Dell'Olmo P. Multi-objective management in freight logistics: increasing capacity, service level and safety with optimization algorithms. Springer, 2008.
33. Katz D, Kompella K, Yeung D. Traffic engineering (te) extensions to ospf version 2. Technical Report, IETF RFC 36302003.

34. Coltun R. The ospf opaque lsa option. Technical Report, IETF RFC 23281998.

35. Moy J. Ospf version 2. Technical Report, IETF RFC 21781997.

36. OPNET Technologies I. The opnet network simulator. URL http: / / www . opnet. com.

37. Inkret R, Lackovic M, Mikac B. Wdm network availability performance analysis for the cost 266 case study topologies. 7th IFIP Working Conference on Optical Network Design and Modelling (ONDM 2003) 2003; :1201-1220.

38. Agency IE. $\mathrm{CO}_{2}$ emissions from fuel combustion highlights 2010. Technical Report, IEA Statistics 2010.

39. Schwartz M. Telecommunication networks: protocols, modeling and analysis, vol. 7. Addison-Wesley Reading, 1987.

\section{AUTHORS' BIOGRAPHIES}

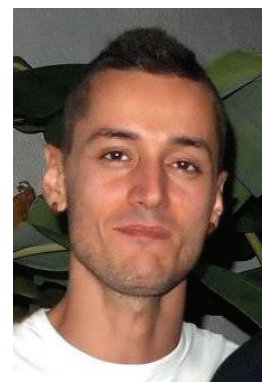

Sergio Ricciardi is a research associate in the Advanced Broadband Communications Center (CCABA) at the Department of Computer Architecture of the Technical University of Catalonia - BarcelonaTech (UPC). He holds a $\mathrm{PhD}$ in Computer Architecture from the UPC and two Masters of Science in Computer Science from the University of Naples Federico II and the UPC. He worked in several national and international projects, such as STRONGEST, SCoPE, COST IC0804 and the CERN LHC ATLAS experiment. He is currently involved in LIGHTNESS, EULER and DOMINO projects. His research interests are mainly focused on energy-aware RWA algorithms and protocols for telecommunication networks and energy-oriented optimizations for grid and cloud computing. 


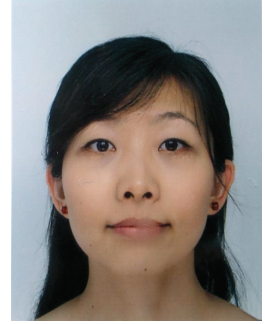

Jiayuan Wang received a B.Eng degree in Communication Engineering in Tianjin University, China, in 2007, and M.Sc in Telecommunications in Technical University of Denmark in 2010. She currently is working as a $\mathrm{PhD}$ student in Technical University of Denmark (DTU). Her research interests include transport network technologies, i.e. MPLS-TP, PBB-TE; energy efficiency in optical core networks; network control and management; network architecture; routing optimization; network modelling and simulation.

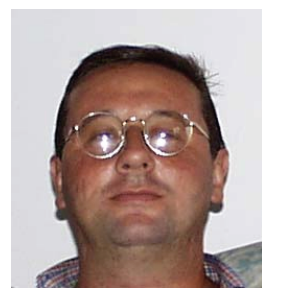

Francesco Palmieri is an assistant professor at the Engineering Faculty of the Second University of Napoli, Italy. His major research interests concern high performance networking protocols and architectures, routing algorithms and network security. Since 1989, he has worked for several international companies on networking-related projects and, starting from 1997, and until 2010 he has been the Director of the telecommunication and networking division of the Federico II University, in Napoli, Italy. He has been closely involved with the development of the Internet in Italy as a senior member of the Technical-Scientific Advisory Committee and of the CSIRT of the Italian NREN GARR. He has published a significant number of papers in leading technical journals and conferences and given many invited talks and keynote speeches.

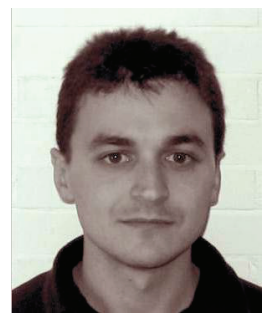

Davide Careglio received the M.Sc. and Ph.D. degrees in telecommunications engineering both from the Technical University of Catalonia - BarcelonaTech (UPC), Barcelona, Spain, in 2000 and 2005, respectively, and the Laurea degree in electrical engineering from Politecnico di Torino, Turin, Italy, in 2001. He is currently an Associate Professor in the Department of Computer Architecture at UPC. Since 2000, he has been a Staff Member of the Advanced Broadband Communication Center. His research interests include networking protocols with emphasis on optical switching technologies, and algorithms and protocols for traffic engineering and QoS provisioning. He has participated in many European and national projects in the field of optical networking and green communication.

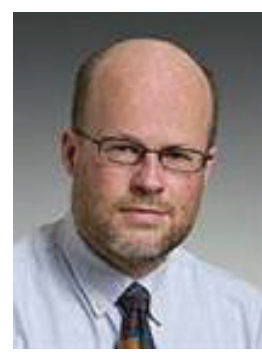

Lars Dittman was born in 1962 and received the M.Sc. EE and Ph.D. from the Technical University of Denmark in 1988 and 1994, and he is currently Professor at the University within the area of integrated network. Lars Dittman has since January 1999 been heading the network competence area (covering both optical and electrical networks) within the Research Center COM at the Technical University of Denmark and was prior to that responsible for electronic switching and ATM networks at the Center for Broadband Telecommunication. Lars Dittman has been involved in a number of EU and national projects working on packet switching, in which he has contributed with traffic and protocol analysis as well as design of electronic IC's and systems. 